() Institute of Mathematical Statistics, 2018

\title{
On the Relationship between the Theory of Cointegration and the Theory of Phase Synchronization
}

\author{
Rainer Dahlhaus, István Z. Kiss and Jan C. Neddermeyer
}

\begin{abstract}
The theory of cointegration has been a leading theory in econometrics with powerful applications to macroeconomics during the last decades. On the other hand, the theory of phase synchronization for weakly coupled complex oscillators has been one of the leading theories in physics for many years with many applications to different areas of science. For example, in neuroscience phase synchronization is regarded as essential for functional coupling of different brain regions. In an abstract sense, both theories describe the dynamic fluctuation around some equilibrium. In this paper, we point out that there exists a very close connection between both theories. Apart from phase jumps, a stochastic version of the Kuramoto equations can be approximated by a cointegrated system of difference equations. As one consequence, the rich theory on statistical inference for cointegrated systems can immediately be applied for statistical inference on phase synchronization based on empirical data. This includes tests for phase synchronization, tests for unidirectional coupling and the identification of the equilibrium from data including phase shifts. We study two examples on a unidirectionally coupled Rössler-Lorenz system and on electrochemical oscillators. The methods from cointegration may also be used to investigate phase synchronization in complex networks. Conversely, there are many interesting results on phase synchronization which may inspire new research on cointegration.
\end{abstract}

Key words and phrases: Cointegration, phase synchronization, weakly coupled oscillators, driver response relationship, Rössler-Lorenz system.

\section{INTRODUCTION}

Phase synchronization has a long history dating back to 1665 , where the mathematician and physicist $\mathrm{C}$. Huygens discovered synchronization of two pendulum clocks suspended close to each other on the same wooden beam. From that time on, the phenomenon

Rainer Dahlhaus is Professor, Institute of Applied Mathematics, Heidelberg University, 69120 Heidelberg, Germany (e-mail: dahlhaus@ statlab.uni-heidelberg.de). István Z. Kiss is Arts and Sciences Associate Professor of Chemistry, Department of Chemistry, Saint Louis University, 3501 Laclede Ave. St. Louis, Missouri 63103, USA (e-mail: izkiss@slu.edu).Jan C. Neddermeyer is Quantitative Analyst, DZ BANK AG, 60325 Frankfurt am Main, Germany (e-mail: jc@neddermeyer.net). got more and more into the focus of scientists. During the last decades, the behavior of two or several interacting oscillators has been intensively studied in the physics literature-in particular, in the context of nonlinear dynamical systems (cf. Pecora and Carroll, 1990, Kocarev and Parlitz, 1996, Rosenblum, Pikovsky and Kurths, 1996, Boccaletti, Pecora and Pelaez, 2001, Boccaletti et al., 2002; see also the monographs Pikovsky, Rosenblum and Kurths, 2001a, Osipov, Kurths and Zhou, 2007). In contrast to other types of synchronization, phase synchronization purely depends on the phases of self-sustained weakly-coupled oscillators while the amplitudes may even be empirically uncorrelated. Weak coupling means that the phases of all oscillators may be subject to individual disturbances and the whole system adjusts itself af- 
terward. Thus phase synchronization is regarded as a complex dynamical process rather than a fixed state.

The phenomena of phase synchronization has been found experimentally in many fields, for example, electrical circuits in Pujol-Pere et al. (2003), Baptista et al. (2003), lasers in DeShazer et al. (2001), electrochemistry in Kiss and Hudson (2001, 2002), biological systems in Elson et al. (1998), Tass et al. (1998), population dynamics in Blasius, Huppert and Stone (1999), between El Niño and the Indian monsoon in Maraun and Kurths (2005) and even tennis in Palut and Zanone (2005). Since phase synchronization purely depends on the phases of the oscillators, it can also be detected between oscillators which are of a different type. A practical example is the phase synchronization of the cardiac and respiratory system in Stefanovska (2002), Stefanovska et al. (2000) or between maternal breathing and the fetal-maternal heart rate coordination in Van Leeuwen et al. (2009).

In neuroscience, phase synchronization is regarded as essential for functional coupling of different brain regions. Single neuronal activity, coupled to an ensemble of oscillatory neuronal activity through the phase, enables the cells to transmit their information content long-range across different cortical areas. To cite from the abstract of Fell and Axmacher (2011): "In recent years, studies ranging from single-unit recordings in animals to EEG and MEG studies in humans have demonstrated the pivotal role of phase synchronization in memory processes. Phase synchronizationhere referring to the synchronization of oscillatory phases between different brain regions-supports both working memory and long-term memory and acts by facilitating neural communication and by promoting neural plasticity." Colgin and Moser (2010) discuss mechanisms of gamma oscillations in the hippocampus and the functional role of the synchronization of such oscillations in several key hippocampal operations, including cell grouping, dynamic routing and memory. In Womelsdorf et al. (2007), synchronization in the gamma band is investigated, and it is discovered that the mutual influence among neuronal groups depends on the phase relation between rhythmic activities within the groups. Furthermore, the pattern of synchronization is related to the pattern of neuronal interactions. Further reviews on phase synchronization in neuroscience are Engel, Fries and Singer (2001), Varela et al. (2001) and David et al. (2003).

To study the synchronization of a larger population of interacting units theoretically, for example, fireflies flashing or crickets chirping at the same time, Winfree
(1967) studied the nonlinear dynamics of a large population of weakly coupled oscillators with intrinsic frequencies that were distributed according to some prescribed probability distribution. He also ignored the amplitude and considered phase oscillators and worked with a mean field model. Kuramoto $(1975,1984)$ introduced a sound mathematical model to describe this phenomenology leading to a theoretical treatment of the mean field approach. He also studied the limit behavior when the number of oscillators tends to infinity. This model has been used since then in different forms to discuss theoretically phase synchronization in a population of weakly coupled oscillators. For example, the onset of synchronization is discussed in this framework in Strogatz (2000).

Although some stochastic methods are used to analyze phase synchronization (e.g., the spectral coherence), there hardly exist any stochastic models for the dynamics of synchronized phase processes. Establishment of experiment-adapted stochastic phase models would be an important task to estimate the dynamics of the phases. Specific outcomes could be statistical tests for phase synchronization, the presence of unidirectional coupling and the identification of the equilibrium from data including phase shifts.

In this paper, we propose that the theory of cointegration provides a good stochastic model for phase synchronization and therefore is a good framework for investigating these questions. In the method, cointegration is not used directly as a model for the oscillators, but rather as a model for the phase processes driving the oscillators. Using the phase processes as the key element for describing oscillators has been standard in physics for many years. On the other hand, this approach has never been used to our knowledge in statistics or econometrics to model oscillators. Here, oscillators have typically been investigated with spectral methods such as Fourier or wavelet transforms. Furthermore, statisticians focused on building models directly for the oscillating processes instead of the phases.

The theory of cointegration may be used in phase synchronization both for theoretical studies and for identifying unknown systems based on empirical data. The link may also be of importance in the other direction in that the known results on phase synchronization may lead to new insights or stimulate research in cointegration. The common ground of both theories is that two or several processes fluctuate randomly around some equilibrium. 
The concept of cointegration was introduced by Granger (1981) for the joint modeling of several macroeconomic variables over time. In 2003, he received the Nobel Prize for his discovery jointly with Engle. The aim of the theory is to model common stochastic trends, for example, of income and consumption where the short-run dynamics is affected by random disturbances and the long-run dynamics is restricted by economic equilibrium relationships. Other examples are the exchange rates and the price levels, the short and long-term interest rates and the prices on spot and future markets.

Since its introduction the theory of cointegration has been developed by many researchers and it has become a leading theory in econometrics with many applications in macroeconomics and beyond; for references, see the monographs Banerjee et al. (1993), Johansen (1995), Engle and White (1999), Lütkepohl (2005) and Juselius (2006) among many others. Phillips (1991) and Kessler and Rahbek (2001) have developed the theory for continuous time diffusion processes.

We now give a heuristic and elementary argument why the setting of cointegration is of importance for phase synchronization: Suppose we observe $y_{t}^{(i)}=$ $A_{i} \cos \left(\phi_{t}^{(i)}\right)+\varepsilon_{t}^{(i)}$ for $i=1,2$ and $t=1, \ldots, T$ with phase processes $\phi_{t}^{(i)}$ (alternatively we may just have the phases $\phi_{t}^{(i)}$ from some oscillators calculated by means of the Hilbert transform or some other method). A naive model for a random phase could be $\phi_{t}=$ $\omega t+\phi_{0}+\delta_{t}$ with some random error $\delta_{t}$ which could be a white noise process or some stationary process. This model had the drawback that it fluctuates around the deterministic linear phase $\omega t+\phi_{0}$ and would therefore only be adequate if some external force or constraint would keep the phase close to this linear phase. Instead a better model for most situations is the corresponding model for the phase increments $\Delta \phi_{t}=$ $\phi_{t}-\phi_{t-1}=\omega+\delta_{t}$ where $\delta_{t}$ is a stationary process with mean zero and positive correlation (in a more refined model one would in addition request positivity of the phase increments; cf. Appendix A). In the simplest case where $\delta_{t}$ is i.i.d. Gaussian, this means that $\phi_{t}=\omega t+\phi_{0}+\sum_{s=1}^{t} \delta_{s}$ has the same distribution as a Brownian motion with drift. In this case, $\operatorname{var}\left(\phi_{t}\right) \sim t$ which means that the phase may depart substantially from $\phi_{t}=\omega t+\phi_{0}$ for large $t$. This feature remains if the process $\delta_{t}$ is stationary with mean 0 . Such a process $\phi_{t}$ is called an integrated process.

If we look at two processes with synchronized phases, then both phases may evolve like a Brownian motion with drift; however, the difference $\phi_{t}^{(1)}-\phi_{t}^{(2)}$ should stay relatively small; in particular, it should not explode. A proper model therefore is that these differences follow a stationary process. This is exactly the concept of cointegration: both phase-processes are integrated but the difference remains stationary. The heuristics presented here is formalized in Appendix A.

The paper is organized as follows. Section 2 provides a brief introduction to phase synchronization with focus on testing for phase synchronization and the $\mathrm{Ku}$ ramoto model for interacting oscillators. We point out that a cointegrated system arises as the solution of a system of stochastic difference equations which is similar to the Kuramoto equations. In Section 3, we give a brief introduction to the theory of cointegration and review some tests for cointegration. Section 4 describes the use of cointegration for the statistical analysis of phase synchronization. In particular, we give our definition of stochastic phase synchronization. In Section 5, we present a simulation showing the application of the proposed method to a coupled Rössler-Lorenz system, and an experimental example of electrochemical oscillators. Conclusions and an outlook are given in Section 6 followed by the definition of VEC-state oscillators in Appendix A and some computational and modeling aspects in Appendix B.

\section{PHASE SYNCHRONIZATION AND THE KURAMOTO MODEL FOR INTERACTING OSCILLATORS}

\subsection{Phase Synchronization of Weakly Coupled Oscillators}

The starting point of phase synchronization is the definition of the phase $\phi_{t}$ of an oscillator. For chaotic phase synchronization, the phase often is defined via the Hilbert transform of the signal. If the projection of the attractor on some plane has only one rotation center (as in Figure 2), then the phase can be defined by the rotation angle around this center. The Hilbert phase of a signal $y_{t}$ is defined through the analytic signal $\zeta_{t}$ given by $\zeta_{t}=y_{t}+i y_{t}^{H}=a_{t} \exp \left(i \phi_{t}\right)$. The imaginary part of $\zeta_{t}$ is obtained using the Hilbert transform; for a definition in the continuous case cf. Pikovsky et al. (1997), Appendix A.2, and for a definition in the discrete time case, cf. Brillinger (2001), Section 2.7. $a_{t}$ is the amplitude, and the phase $\phi_{t}$ is computed through

$$
\phi_{t}=\arctan \frac{y_{t}^{H}}{y_{t}} .
$$


Alternatively, one may look at stochastic systems, for example, of the form

$$
y_{t}=a_{t} \cos \left(\phi_{t}\right)+\varepsilon_{t}, \quad t \in \mathbb{Z}
$$

or even at systems like

$$
y_{t}=a_{t} f\left(\phi_{t}\right)+\varepsilon_{t}, \quad t \in \mathbb{Z},
$$

where $f$ is a $2 \pi$ periodic real valued function representing the oscillation pattern. Here, the $\phi_{t}$ may be determined by a particle filter (Dahlhaus et al., 2017). The latter model may, for example, be used for ECG signals. Further methods for phase estimation are based on the wavelet transform (Grossmann, Kronland-Martinet and Morlet, 1989), or on a local periodogram (Hannan, 1973, Paraschakis and Dahlhaus, 2012).

In the next step, two weakly coupled oscillators with phases $\phi_{t}^{(1)}$ and $\phi_{t}^{(2)}$ are called $m: n$ phase synchronized if there exists some constant $\Phi$ (phase shift) and some small $\delta>0$ such that

$$
\left|\left(m \phi_{t}^{(1)}-n \phi_{t}^{(2)}-\Phi\right) \bmod 2 \pi\right|<\delta
$$

holds for all $t . m$ and $n$ are integers which are usually known in a practical application. The idea behind this definition is that the (rescaled) phases "stay together" and do not move arbitrarily far from each other. A standard statistic for testing the hypothesis "no phase synchronization" is the phase synchronization index (Mormann et al., 2000, Quian Quiroga, Kreuz and Grassberger, 2002b) defined through

$$
\hat{R}^{2}=\left|\frac{1}{T} \sum_{t=1}^{T} \exp \left\{i\left(m \phi_{t}^{(1)}-n \phi_{t}^{(2)}\right)\right\}\right|^{2} .
$$

The synchronization index has values between 0 and 1 where values close to one indicate phase synchronization. A value close to 1 is obtained for an almost constant phase difference of $\left|m \phi_{t}^{(1)}-n \phi_{t}^{(2)}\right|$, which can occur even for two nonidentical chaotic oscillators (Rosenblum, Pikovsky and Kurths, 1996). $\hat{R}^{2}$ has often been used for testing phase synchronization and there exist several articles where the distribution of $\hat{R}^{2}$ or other test statistics is approximated by different methods, for example, with surrogate data. The most advanced approach from a statistical perspective is Schelter et al. (2007) where the distribution of $\hat{R}^{2}$ under the null hypothesis is approximated by using two independent stochastic processes. Reviews and comparisons of various phase synchronization measures and tests can be found in Quian Quiroga, Kreuz and
Grassberger (2002b), Allefeld and Kurths (2004b) and Schelter et al. (2007).

Despite of these results, the situation is not satisfying from a statistical perspective, because there usually does not exist a clearly defined population quantity corresponding to $\hat{R}^{2}$. We show in this paper that cointegrated systems provide a statistical framework with which questions like existence of synchronization and unidirectional coupling can be investigated.

\subsection{Cointegration as a Stochastic Kuramoto-Type Model}

As mentioned in the Introduction, Kuramoto (1975, 1984) introduced a mathematical model to describe the synchronization of a larger population of interacting units. A simple version of the Kuramoto model for the phases of $d$ oscillators is

$$
\begin{aligned}
\dot{\phi}^{(i)} & =\omega_{i}+\frac{K}{d} \sum_{j=1}^{d} \sin \left(\phi^{(j)}-\phi^{(i)}\right) \\
(i & =1, \ldots, d) .
\end{aligned}
$$

Allefeld and Kurths (2004a) discuss the following stochastic generalization of the model:

$$
\begin{gathered}
\dot{\phi}^{(i)}=\omega_{i}+\sum_{j=1}^{d} k_{i j} \sin \left(\phi^{(j)}-\phi^{(i)}\right)+\eta_{i} \\
(i=1, \ldots, d)
\end{gathered}
$$

where the $\eta_{i}$ are taken to be independent Gaussian white noise variables.

There is a striking similarity of this model to cointegration: For $\phi^{(j)}-\phi^{(i)}$ small, we can approximate $\sin \left(\phi^{(j)}-\phi^{(i)}\right)$ by $\phi^{(j)}-\phi^{(i)}$ leading to the discretized approximation for the vector $\phi_{t}=\left(\phi_{t}^{(1)}, \ldots, \phi_{t}^{(d)}\right)^{\prime}$, $\omega=\left(\omega_{1}, \ldots, \omega_{d}\right)^{\prime}$

$$
\Delta \phi_{t}=\omega+\Pi \phi_{t-1}+\eta_{t}
$$

with $\Pi=K-\operatorname{diag}\left(\sum_{j=1}^{d} k_{1 j}, \ldots, \sum_{j=1}^{d} k_{d j}\right)$ where $K=\left(k_{i j}\right)_{i, j=1, \ldots, d}$. This is exactly the representation (9) from below with $p=1$ and $\omega=\mu$, that is, the cointegrated system (11), (10) with $p=1$ is exactly the solution of the stochastic difference equations (7). We mention that cointegration requires the matrix $\Pi$ to be of reduced rank which is fulfilled in this case since the columns sum up to 0 .

Being a bit more specific at this point gives us a deeper insight into the relevance of cointegration theory for phase synchronization in networks: The above 
$\Pi$ can be written in the form $\Pi=\alpha \beta^{\prime}$ with $d \times r$ matrices $\alpha$ and $\beta$ of full rank $r$ where (in this case) $r=d-1$, namely

$$
\beta^{\prime}=\left(\begin{array}{c}
\beta_{1}^{\prime} \\
\vdots \\
\beta_{r}^{\prime}
\end{array}\right)=\left(\begin{array}{ccccc}
1 & 0 & \cdots & 0 & -1 \\
0 & 1 & \cdots & 0 & -1 \\
\vdots & & \ddots & & \vdots \\
0 & 0 & \cdots & 1 & -1
\end{array}\right)
$$

and $\alpha$ consisting of the first $d-1$ columns of $\Pi$ (only for this specific $\beta$ ). The $\beta_{j}^{\prime} \phi_{t}=0$ are obviously the $d-1$ phase synchronization relations $\phi_{t}^{(1)}=\cdots=$ $\phi_{t}^{(d)}$. The matrix $\alpha$ is sometimes called adjustment or loading matrix.

In the following, we apply the concept of cointegration to phase synchronization. For the model (7), this means testing for cointegration (phase synchronization) via determining (testing) $r=\operatorname{rank} \Pi$. This results in the reduced rank representation $\Pi=\alpha \beta^{\prime}$ with $d \times r$-matrices $\alpha$ and $\beta$ of full rank $r$. After the parameters $\alpha$ and $\beta$ have been estimated, $\beta^{\prime} \phi_{t}$ are the $r$ cointegration (phase synchronization) relations and $\alpha$ gives the intensities with which deviations from the equilibrium lead to corrections. In particular, $\alpha$ can be tested for unidirectional coupling.

The use of the cointegration model (7) for phase synchronization has several benefits: The model can be fitted in both situations (phase synchronization/nonphase synchronization) to the data and the hypothesis of phase synchronization can be tested on the matrix $\Pi$, for example, by looking at the fitted model. In addition, one is able to identify the parameters from real data, and to conclude to the phase synchronization relations, unidirectional coupling, etc. This can easily be done by using the large number of existing tools for cointegration.

On the other hand, it is very important to keep the difference between the two models in mind: The major difference is that the Kuramoto model (6) has the equilibrium $\phi^{(j)}-\phi^{(i)} \equiv 0 \bmod 2 \pi$ while the cointegration model (7) has the equilibrium $\phi^{(j)}-\phi^{(i)}=0$. In case of (say) $\pi / 2<\phi^{(j)}-\phi^{(i)}<\pi$, the Kuramoto model has a force to the "equilibrium" $\phi^{(j)}-\phi^{(i)}=2 \pi$ while the cointegration model still has a force to $\phi^{(j)}-\phi^{(i)}=$ 0 . This means that the Kuramoto model allows for phase jumps of one of the two processes while the cointegration model does not. One may term the former "weak synchronization" and the latter "strong synchronization." Examples are the processes with $\epsilon=$ 9.6, 10.2, 11.0, 11.1 in Section 5.1 (Figure 4 and Figure 5) which exhibit phase jumps.

\section{THE CONCEPT OF COINTEGRATION}

\subsection{Cointegration and the Granger Representation Theorem}

We now briefly review a small part of the theory of cointegration (for more details see, for example, the monographs Johansen, 1995, Lütkepohl, 2005, Juselius, 2006) — the last one containing a more applied view). As it is usual in many papers, we restrict ourselves to vector autoregressive (VAR-) processes

$$
X_{t}=A_{1} X_{t-1}+\cdots+A_{k} X_{t-p}+\mu+\eta_{t},
$$

where the coefficients $A_{i}$ are $d \times d$-matrices, $\mu=$ $\left(\mu_{1}, \ldots, \mu_{d}\right)^{\prime}$ and the innovations $\eta_{t}$ are i.i.d. with mean 0 and positive definite covariance matrix $\Omega_{\eta}$. Let $A(z):=I_{d}-A_{1} z-\cdots-A_{p} z^{p}$ be the characteristic polynomial. We restrict ourselves to VAR-processes with roots outside the unit circle or equal to 1 , that is, where $\operatorname{det}(A(z))=0$ implies $|z|>1$ or $z=1$.

We call a process integrated (of order 1 - I(1) for short) if $X_{t}$ is not stationary and the first-order difference $\Delta X_{t}=X_{t}-X_{t-1}$ is stationary. We call a $d$ dimensional process $X_{t}$ cointegrated if each univariate series is integrated and some linear combination $\beta^{\prime} X_{t}$ (with an $r \times d$-matrix $\beta$ of rank $r$ and $0<r<d$ ) is stationary (these definitions simplify the issue-for thorough definitions we refer to the above monographs). $\beta$ is known as the cointegrating vector. It specifies the long-term relationship between the involved univariate series. The most relevant example for phase synchronization of 2 oscillators is $d=2$ and $\beta=(1,-1)^{\prime}$ : $\beta^{\prime} X_{t}$ fluctuates stationary around a long run mean, that is, it is bounded in probability.

With some straightforward calculations we can transform the above representation to

$$
\Delta X_{t}=\Pi X_{t-1}+\sum_{i=1}^{p-1} \Gamma_{i} \Delta X_{t-i}+\mu+\eta_{t},
$$

where

$$
\Pi:=-\left(I_{d}-A_{1}-\cdots-A_{p}\right)
$$

and

$$
\Gamma_{i}:=-\left(A_{i+1}+\cdots+A_{p}\right)
$$

for $i=1, \ldots, p-1$. If the process $X_{t}$ is not stationary (in particular if it is cointegrated) then $\operatorname{det}(\Pi)=$ $(-1)^{d} \operatorname{det}(A(1))=0$. Thus $\Pi$ is singular with rank $r<d$ and it can be decomposed to $\Pi=\alpha \beta^{\prime}$ with $d \times r$ matrices $\alpha$ and $\beta$ of full rank $r$.

Since the process is $I(1)$, the first-order difference $\Delta X_{t}$ is stationary, and the representation (9) implies 
that also $\Pi X_{t-1}=\alpha \beta^{\prime} X_{t-1}$ is stationary. Multiplying this with $\left(\alpha^{\prime} \alpha\right)^{-1} \alpha^{\prime}$ implies that $\beta^{\prime} X_{t-1}$ is stationary, that is, each component of $\beta^{\prime} X_{t-1}$ is a cointegrating relation. The corresponding representation

$$
\Delta X_{t}=\alpha \beta^{\prime} X_{t-1}+\sum_{i=1}^{p-1} \Gamma_{i} \Delta X_{t-i}+\mu+\eta_{t}
$$

is called vector error correction model (VEC-model or VEC-representation). It shows that whenever the process moves away from the equilibrium $\beta^{\prime} X_{t-1}=\beta_{0}$ (for the definition of $\beta_{0}$ see below) it is pulled back to the equilibrium with the forces $\alpha$ (see the end of Section 5.1 for an illustrative example).

The Granger representation theorem (Engle and Granger, 1987, Johansen, 1991) now gives another useful representation of the process. Under the above conditions, the process has the moving average representation

$$
X_{t}=C \sum_{i=1}^{t} \eta_{i}+C \mu t+C^{*}(L)\left(\eta_{t}+\mu\right)+X_{0}^{*},
$$

where

$$
C=\beta_{\perp}\left[\alpha_{\perp}^{\prime} \Gamma \beta_{\perp}\right]^{-1} \alpha_{\perp}^{\prime}
$$

with $\quad \Gamma:=I_{p}-\sum_{i=1}^{p-1} \Gamma_{i} . \quad C^{*}(L)\left(\eta_{t}+\mu\right)=$ $\sum_{j=0}^{\infty} C_{j}^{*}\left(\eta_{t-j}+\mu\right)$ is a stationary process and $X_{0}^{*}$ contains initial values with $\beta^{\prime} X_{0}^{*}=0 . \alpha_{\perp}$ denotes an orthogonal complement of $\alpha$, that is, $\alpha_{\perp}$ is an $d \times(d-r)$ matrix of rank $d-r$ with $\alpha_{\perp}^{\prime} \alpha=0$ (the same for $\beta_{\perp}$ ).

The representation (11) shows that the $d$-dimensional process is driven by $d-r I(1)$ components and $r$ stationary components. The first term on the right hand side consists of $d$ random walks $\sum_{i=1}^{t} \eta_{i}$ which are multiplied by a matrix of rank $d-r$ denoted by $C$. Thus, there are actually $d-r$ stochastic trends driving the system. On the contrary, the representation (10) [or (14)] shows how the process is pulled back to the equilibrium if deviations occur. This is illustrated by the example at the end of Section 5.1.

Of special importance for phase synchronization is a decomposition $\mu=\Gamma \omega_{0}-\alpha \beta_{0}$ where $\omega_{0}$ is a trendterm and $\beta_{0}$ is a constant belonging to the error correction equation: Taking expectations in (11), we obtain because of $\mathbf{E} \eta_{t}=0$

$$
\mathbf{E} X_{t}=C \mu t+C^{*}(L) \mu+\mathbf{E} X_{0}^{*}
$$

and, therefore, $\omega_{0}:=\mathbf{E} \Delta X_{t}=C \mu$. Since $\Delta X_{t}$ is stationary, we obtain from (10)

$$
\alpha \mathbf{E}\left(\beta^{\prime} X_{t-1}\right)=\mathbf{E}\left(\Delta X_{t}\right)-\sum_{i=1}^{p-1} \Gamma_{i} \mathbf{E}\left(\Delta X_{t-i}\right)-\mu
$$

$$
=\left[\Gamma C-I_{d}\right] \mu .
$$

Let $\bar{\alpha}:=\alpha\left(\alpha^{\prime} \alpha\right)^{-1}$. Since $\bar{\alpha}^{\prime} \alpha=I_{r}$, we have $\mathbf{E}\left(\beta^{\prime} X_{t-1}\right)=\bar{\alpha}^{\prime}\left[\Gamma C-I_{d}\right] \mu=: \beta_{0}$.

Obviously, we have $\mu=\Gamma C \mu-\left[\Gamma C-I_{d}\right] \mu=$ $\Gamma \omega_{0}-\alpha \beta_{0}$. Therefore, we obtain the modified VECrepresentation

$$
\begin{aligned}
\left(\Delta X_{t}-\omega_{0}\right)= & \alpha\left(\beta^{\prime} X_{t-1}-\beta_{0}\right) \\
& +\sum_{i=1}^{p-1} \Gamma_{i}\left(\Delta X_{t-i}-\omega_{0}\right)+\eta_{t} .
\end{aligned}
$$

This means that the "true" cointegration relation describing the equilibrium is $\beta^{\prime} X_{t-1}-\beta_{0}=0$. If the process deviates at time $t-1$ from this relation, it is pulled in the next step with force $\alpha$ back towards this equilibrium. (11) and (13) show that $\omega_{0}=C \mu$ is the drift-vector of the process, that is the process $X_{t}$ has a deterministic trend $\omega_{0} t$. The intercept terms are contained in $X_{0}^{*}$ in (11).

Below we use this model as a stochastic model for phase synchronization. In the case $d=2$, the situation is even more intuitive: If the system is cointegrated (i.e., $r=1$ ), we only have a one-dimensional cointegration relation $\beta^{\prime} X_{t-1}-\beta_{0}=0$ and the drift vector $\omega_{0}$ has the same direction as the random walk part $C \sum_{i=1}^{t} \eta_{i}$ (namely $\beta_{\perp}$ ). We discuss this simpler and more intuitive case in Section 5.1 with a specific example.

To keep the situation simple, we first restrict ourselves to the case $p=2$. In this case, the VECrepresentation takes the form (now replacing $X_{t}$ by $\phi_{t}$ )

$$
\begin{aligned}
\Delta \phi_{t}^{(1)}= & \alpha_{1}\left(\phi_{t-1}^{(1)}-\beta_{2} \phi_{t-1}^{(2)}\right) \\
& +\gamma_{1} \Delta \phi_{t-1}^{(1)}+\delta_{1} \Delta \phi_{t-1}^{(2)}+\mu_{1}+\eta_{t}^{(1)}, \\
\Delta \phi_{t}^{(2)}= & \alpha_{2}\left(\phi_{t-1}^{(1)}-\beta_{2} \phi_{t-1}^{(2)}\right) \\
& +\gamma_{2} \Delta \phi_{t-1}^{(2)}+\delta_{2} \Delta \phi_{t-1}^{(1)}+\mu_{2}+\eta_{t}^{(2)},
\end{aligned}
$$

where we have assumed for the cointegrating vector $\beta=\left(1,-\beta_{2}\right)^{\prime}$ for identifiability. In that case, also $\alpha_{1}$ and $\alpha_{2}$ are identifiable.

If the model is used for phase synchronization $\beta_{2}$ usually is known (in the $1: 1$ synchronization case we have $\beta_{2}=1 ; \beta_{0}$ then is the mean phase difference in the equilibrium). We expect that in many cases the reduced system with $\delta_{1}=\delta_{2}=0$ will suffice to describe the joint phase dynamics. However, the parameters $\gamma_{1}$, $\gamma_{2}$ are needed since successive increments usually are correlated. 
In the reduced system where $\delta_{1}=\delta_{2}=0$, error correction toward the equilibrium is done solely by the terms with coefficients $\alpha_{1}$ and $\alpha_{2}$. Of special importance are the cases $\alpha_{1}<0, \alpha_{2}=0$ and $\alpha_{1}=0, \alpha_{2}>0$ meaning that there is unidirectional coupling; see Section 5.1 for an illustrative example (in the nonreduced system also the higher order terms contribute to error correction - cf. Johansen, 1995, Exercise 4.3). This allows for testing whether there is an unidirectional driver-response relationship between the phases.

A general state space model is discussed in Appendix A. There also the positivity of the phase increments is discussed.

\subsection{Cointegration Testing and Model Fitting}

In order to test for phase synchronization and for unidirectional driver-response relationships, we apply tests for cointegration. Two cases need to be distinguished: First, the case where the cointegrating vector $\beta$ is known and we want to test for a specific cointegrating relationship. This may be the standard case for phase synchronization of two oscillators where the $m: n$ synchronization relation as in (3) is often clear from prior knowledge or eye-inspection.

The other case is the case of unknown $\beta$. For example, in systems of higher dimension it may be clear that the whole system is "somehow" $1: 1$ synchronized but it usually is not clear at all how the synchronization "propagates" through the network. In that case, the task is to test for the rank of $\Pi$ in (9) and to determine the factorization $\Pi=\alpha \beta^{\prime}$ as well as the vector $\beta_{0}$ leading to the phase synchronization relations, the mean phase shift and the matrix $\alpha$ representing the strength and the direction of coupling.

We start with the case of unknown $\beta$ leading to the likelihood theory of Johansen (1995) and to his likelihood ratio test. Additional information on this case is provided in Section 4. We then discuss the augmented unit root test by Dickey and Fuller $(1979,1981)$. We mention that there exist several other tests such as a test based on residuals by Phillips and Ouliaris (1990) and Lagrange multiplier tests (Saikkonen and Lütkepohl, 2000).

Johansen's likelihood theory and rank test. The likelihood theory for cointegrated systems is well developed - the most famous result being Johansen's likelihood ratio test (LR-test) for the cointegration rank, that is, for the determination of $r=\operatorname{rank}(\Pi)$ in (9) and the corresponding decomposition $\Pi=\alpha \beta^{\prime}$ with $\alpha$ and $\beta$ being $d \times r$-matrices of rank $r$. Thus a major advantage of the Johansen procedure is that it can simultaneously identify multiple cointegration relations in multivariate time series. The situation is much more challenging than for the classical likelihood ratio test since the integrated processes require a different asymptotic theory leading, for example, to a nonstandard limit distribution of the likelihood ratio test.

We briefly sketch part of the results, for a more detailed discussion, cf. Chapters 7 and 8 in Juselius (2006). Note that in our case $\mu$ in (9), (10) plays an important role since it leads to the phase lag $\beta_{0}$ and the deterministic drift term $\omega_{0} t$ of the phases, while the additional term $\mu_{1} t$ often discussed in the cointegration literature (leading to quadratic drift) is not needed in this context (i.e., the situation we consider is Case 3 in Juselius, 2006).

Suppose now we want to calculate the maximum likelihood estimates for $\alpha$ and $\beta$ in the system (10). In the Gaussian case, the maximum likelihood estimates are essentially the same as least squares estimates. In the first step, $\mu$ and the $\Delta X_{t-i}$ are removed by regressing them on $\Delta X_{t}$ and $X_{t-1}$. If we denote the residuals by $\widetilde{\Delta X}_{t}$ and $\widetilde{X_{t-1}}$, we obtain the "concentrated model"

$$
\widetilde{\Delta X_{t}}=\alpha \beta^{\prime} \widetilde{X_{t-1}}+\text { error. }
$$

Now, the optimal value $\hat{\alpha}(\beta)$ is determined for given $\beta$, plugged into this equation, and finally the optimal $\beta$ is determined leading to the maximum of the likelihood. This procedure can be viewed as finding the canonical correlations between $\widetilde{\Delta X}$ and $\widetilde{X_{t-1}}$ (cf. Juselius, 2006, Chapter 8). The resulting squared canonical correlations are denoted by $\hat{\lambda}_{1} \geq \cdots \geq \hat{\lambda}_{d} \geq 0$. The task now is to split the $\hat{\lambda}_{1} \geq \cdots \geq \hat{\lambda}_{d}$ into those $\hat{\lambda}_{1}, \ldots, \hat{\lambda}_{r}$ which are different from zero and which belong to a cointegration relation and those $\hat{\lambda}_{r+1}, \ldots, \hat{\lambda}_{d}$ which are not significantly different from zero (more precisely where the corresponding unknown theoretical values are zero). With view on (11) and the discussion below, $d-r$ is the number of driving "forces" of the trend. In particular, $r$ is the rank of the matrix $\Pi$ we are looking for.

A formal way to determine $r=\operatorname{rank}(\Pi)$ based on the values of $\hat{\lambda}_{1}, \ldots, \hat{\lambda}_{d}$ is the likelihood ratio test, for example, the so-called trace-test where the null hypothesis $\mathcal{H}(r)=\{\operatorname{rank}(\Pi) \leq r\}$ is tested in $\mathcal{H}(d):=$ $\{\operatorname{rank}(\Pi) \leq d\}$ recursively for $r=0, \ldots, d-1$ (this recursion is called "top $\rightarrow$ bottom" procedure with "top" 
meaning a large number of cointegration relations corresponding to a small rank). The test statistic is

$$
\tau_{d-r}:=\operatorname{LR}(\mathcal{H}(r) \mid \mathcal{H}(d))=-T \sum_{i=r+1}^{d} \log \left(1-\hat{\lambda}_{i}\right)
$$

(called LR-test below) and the hypothesis is rejected at a significance level $\alpha$ if $\tau_{d-r}>C_{\alpha}(d-r)$ where $C_{\alpha}(d-r)$ is from Appendix A of Juselius (2006) (Case 3; $p-r=d-r$ ). The procedure stops if the test accepts the hypothesis for some $r$.

Special attention is required for the case $r=d$. This means that there are no stochastic trends and the system in (9) is stationary. In particular, this case does not mean that there are $r$ cointegration relations; see the discussion in the next section.

Augmented Dickey-Fuller unit root test. The Augmented Dickey-Fuller (ADF) test allows one to test for the presence of a unit root in a univariate time series $Y_{t}$. It is based on the regression

$$
\Delta Y_{t}=a+b t+c y_{t-1}+\sum_{j=1}^{p} c_{j} \Delta Y_{t-j}+\epsilon_{t} .
$$

The constant $a$ and the time trend $b t$ are only included if required. For $a \neq 0$ and $b=0$, the ADF-test tests the null hypothesis $c=0$ integrated process with a deterministic trend against the alternative $c<0$ stationarity with a mean. The test statistic is the usual t-ratio given by

$$
\mathrm{DF}_{\tau}=\frac{\hat{c}}{\mathrm{SE}(\hat{c})}
$$

with $\hat{c}$ being the ordinary least squares estimator of $c$ and $\operatorname{SE}(\hat{c})$ being its estimated standard error. The corresponding quantiles can be found in Hamilton (1994) where in our case the standard normal distribution must be used. In case the potential cointegration relation $\beta^{\prime} X_{t}$ is known, the ADF-test provides a simple method for cointegration testing. We then apply the ADF-test to the residuals $Y_{t}=\beta^{\prime} X_{t-1}$. Due to $a \neq 0$, it is not necessary to include $\beta_{0}$.

The ADF-test can also be used to test the hypothesis integrated process versus trend stationarity. An alternative is to use the KPSS-test by Kwiatkowski et al. (1992) where the hypothesis are exchanged. A disadvantage of the ADF-test is the implicit common factor restriction which is imposed when the ADF-test is used. The test loses power if the restriction is not satisfied (Kremers, Ericsson and Dolado, 1992). An alternative to the ADF-test is the Wald test which tests for cointegration via the significance of the adjustment coefficients $\alpha$ (Horvath and Watson, 1995).

\section{THE COINTEGRATION APPROACH TO PHASE SYNCHRONIZATION}

\section{The Cointegration Model for Phase Processes}

We now use the concept of cointegration for a stochastic definition of phase synchronization. The key idea is that in a stochastic context phase synchronization can be described in terms of stationarity of the phase-differences, that is, by cointegration. That is we propose to replace the fixed deterministic bound in (3) by a stochastic bound: The difference may even get large (with small probability) but the fact that the difference is stationary will always force it back to the equilibrium.

Before we give the definition, we stress that here we only want to discuss models for signals whose phase increments can be regarded as stationary-that is where the unwrapped phases are integrated processes with a deterministic linear trend (the stochastic part of the integrated process is sometimes called "stochastic trend"). The case where the deterministic trend is zero will hardly occur in practice; the case where the stochastic trend is zero (i.e., the process is trend stationary) may occur in specific cases; see the discussion below. By integrated, we always mean I(1)-integrated in terms of the cointegration literature.

Definition 1 (Stochastic Phase Synchronization). $d$ oscillators with phase processes $\phi_{t}=\left(\phi_{t}^{(1)}, \ldots\right.$, $\left.\phi_{t}^{(d)}\right)^{\prime}$ are called stochastically phase synchronized of order $r$ with $1 \leq r \leq d-1$, if all processes are integrated and there exist $r$ linearly independent vectors $\beta_{j}$ such that the $\beta_{j}^{\prime} \phi_{t}$ are stationary (i.e., $\phi_{t}$ is cointegrated with rank $r$ ). In the case of the VEC-model (9), this means that $\operatorname{rank}(\Pi)=r$ and $\Pi=\alpha \beta^{\prime}$ as in (10) with $\beta=\left(\beta_{1}, \ldots, \beta_{r}\right)$. The $\beta_{j}^{\prime} \phi_{t}$ are up to a constant and up to some nonidentifiability the phase synchronization relations.

One may also use the term stochastically $\beta$-phase synchronized of order $r$ if the processes are cointegrated of rank $r$ with cointegrating matrix/vector $\beta$.

We now briefly discuss the different orders $r$ of phase synchronization which correspond to the rank of the matrix $\Pi$ in (9):

$r=0$ : If $\operatorname{rank}(\Pi)=0$ there do not exist any cointegration (equilibrium) relations and the phases are integrated processes with a deterministic drift term (which for phase processes usually is different from 0 ) and a stochastic drift term (meaning that the processes are integrated). Since the vector of phase increments follows a VAR-process as in (9) with $\Pi=0$ there is still some 
stochastic dependence of the stochastic drifts terms but this does not lead to synchronization of the phases.

$0<r<d$ : In this case, we are having $r$ phase synchronization relations, meaning that $r$ is a measure for the degree of synchronization. We give some examples:

- if $r=1$, we may have, for example, the relation $\phi_{t}^{(1)}=\phi_{t}^{(2)}$ or, for example, the relation $\sum_{j=1}^{d} \beta^{j} \times$ $\phi_{t}^{(j)}-\beta_{0}=0$ (this form may hardly occur in practise);

- if $r=2$, we may have two (linearly) independent relations such as $\phi_{t}^{(1)}=\phi_{t}^{(2)}$ and $\phi_{t}^{(3)}=\phi_{t}^{(4)}$, or two generalized relations as above;

- if $r=d-1$, we have, for example, the relations $\phi_{t}^{(1)}=\cdots=\phi_{t}^{(d)}$.

Recall that this means that the d-dimensional process is stochastically fluctuating around these $r$ phase synchronization relations with some error correction mechanism as given in (14). The phase synchronization relations are not uniquely determined. This is reflected by the equation $\Pi=\alpha \beta^{\prime}=\alpha \xi^{\prime-1}(\beta \xi)^{\prime}$ with any regular $r \times r$-matrix $\xi$ where now the columns of $(\beta \xi)$ are the phase synchronization relations. Instead of single phase synchronization relations we have a phase synchronization space spanned by $\beta$ which is the row space of $\Pi$ (Johansen, 1991). Testing for the rank of $\Pi$ can be viewed as testing for certain subspaces of the phase synchronization space represented by linear restrictions.

The case $r=d$ is a special and difficult one. $\phi_{t}^{(1)}, \ldots, \phi_{t}^{(d)}$ then no longer are cointegrated and there does not exist any equilibrium around which the processes fluctuate. Equation (11) then means that the $d$ dimensional process is driven by $d-r$ integrated components. Thus in the case $r=d$ no stochastic trend terms remain, which are capable of driving the system; the process in (10) is stationary in this case. Furthermore, the term $\mu$ in (9) does not produce any trend in case $\operatorname{rank}(\Pi)=d$. One may investigate then whether all processes are trend stationary, that is, processes with some intercept $\gamma$ and trend $\delta t$ plus some stationary part. This may be caused by an external "pacemaker" such as some daily cycle.

To determine $r=\operatorname{rank}(\Pi)$, we recommend using Johansen's LR-test described above based on the VECrepresentation (9). It is important to include the parameter $\mu$ since this generates the deterministic trend $\omega_{0}$ and the phase shift $\beta_{0}$ (as described above). If $\beta$ is known, one may use other testing methods such as the ADF-test.

Kammerdiner and Pardalos (2010) and Hirsch, Pardalos and Murphey (2010) also had the idea to use cointegration for phase synchronization and applied this to absence epilepsy data and to the analysis of neural data collected from primates. However, their approach is different to the one presented here since they apply cointegration to the wrapped phases instead of the unwrapped phases. We regard this as not adequate since the wrapped phases can hardly be regarded as realizations of an integrated process. As a consequence, the phase processes in their examples were often tested to be stationary and the rank test for cointegration did often lead to the maximal rank $r=d$ indicating stationarity of the phase processes instead of cointegration of integrated processes.

It is standard in physics to proceed as if the phases were observed directly and to ignore the effect of estimating the phases. The estimation of the phases is usually done by means of the Hilbert-transform on a segment (cf. Section 2.1). We will proceed in the following and in the examples in Section 5.1 in the same way.

\section{Applying the Method to Phase Processes}

We now summarize the main steps of the method.

\section{Determination of the phases:}

Given the original observations of the oscillators, compute the phases processes $\phi_{t}^{(j)}(j=1, \ldots, d)$ by using the Hilbert transform or another method (see Section 2.1). Unwrap the phases prior to the subsequent analysis. For specific models such as (1), (2), other methods may be used.

2. Testing for the rank of $\Pi$ :

Use the VAR-model as in (9) with $\mu \neq 0$ and conduct the Johansen LR-test as described above. We prefer the trace test in the "top $\rightarrow$ bottom"-version. For the choice of the order $p$, see the discussion in Appendix B. It is also possible to test specific $\beta$ or hypothesis about $\beta$ (Johansen, 1995, Chapter 7).

In the situation where the possible phase synchronization relations $\beta^{\prime} \phi_{t}$ are known (in particular for $d=2$ ), one may use instead the ADF-test (with $a \neq 0$ and $b=0$ ) to test for $\beta^{\prime} \phi_{t}$ the null hypothesis integrated process versus stationary process. If the test rejects, we conclude to phase synchronization. In principle, the use of our knowledge about $\beta$ results in a slightly higher power of the test. 


\section{Estimation, interpretation and further testing of} the model:

If not already provided from step 2, fit the model (10) with $\beta$ from step 2 to the data and estimate all coefficients. Check for significance of all coefficients and delete those coefficients which are not significant. Calculate $\omega_{0}$ and $\beta_{0}$ as described above and write the whole model in the form

$$
\begin{aligned}
& \left(\Delta X_{t}-\omega_{0}\right) \\
& \quad=\alpha\left(\beta^{\prime} X_{t-1}-\beta_{0}\right)+\sum_{i=1}^{p-1} \Gamma_{i}\left(\Delta X_{t-i}-\omega_{0}\right)+\eta_{t}
\end{aligned}
$$

with the estimated parameters; see (24). Remember that the parameters $\alpha$ and $\beta$ are not uniquely determined (only the cointegration space is unique). This means that different $\beta$ can be chosen to formulate the same equilibrium relations (see also Juselius, 2006, Section 8.5, "The cointegration rank: a difficult choice"). For testing, the coefficients one can use the t-ratio. In the case of cointegration, this t-ratio follows a standard t-distribution.

\section{Testing for unidirectional coupling:}

An important special case of step 3 is the testing for unidirectional coupling. If phase synchronization is detected, one can test $\alpha$ for the direction of the dependency. For example, in the VEC-model (15), (16) with $\delta_{1}=\delta_{2}=0$, the direction of coupling can be investigated with the adjustment coefficients $\alpha_{1}, \alpha_{2}$. If, for example, a significance test suggests that $\alpha_{1}<0$ and $\alpha_{2}=0$ one can conclude that $\phi_{t}^{(2)}$ influences/corrects $\phi_{t}^{(1)}$ but not the other way round. Thus, $\phi_{t}^{(2)}$ is the driving force of phase synchronization and $\phi_{t}^{(1)}$ is forced to adapt.

Software for performing these steps is discussed in the Appendix B.1.

\section{EXAMPLES}

\subsection{A Coupled Rössler-Lorenz System}

As an example, we analyze with the above methods an unidirectionally coupled Rössler-Lorenz system. We proceed through steps 1-4 above. Computational details and additional modeling aspects can be found in Appendix B.

0. Simulation of the data: The system is defined through an autonomous Rössler attractor with configuration

$$
\dot{x_{1}}=-12\left(y_{1}+z_{1}\right)+w_{1},
$$

$$
\begin{aligned}
& \dot{y_{1}}=12\left(x_{1}+0.2 y_{1}\right), \\
& \dot{z_{1}}=12\left[0.2+z_{1}\left(x_{1}-5.7\right)\right],
\end{aligned}
$$

and a driven Lorenz attractor given by

$$
\begin{aligned}
& \dot{x_{2}}=16\left(y_{2}-x_{2}\right)-\epsilon\left(x_{2}-x_{1}\right)+w_{2}, \\
& \dot{y_{2}}=45.92 x_{2}-y_{2}-x_{2} z_{2}, \\
& \dot{z_{2}}=x_{2} y_{2}-4 z_{2}
\end{aligned}
$$

with system noise variables $w_{1}, w_{2}$ being i.i.d. $\mathcal{N}(0$, $0.15^{2}$ ) distributed similar to the system analyzed in Schelter et al. (2007). The parameters are taken from Guan, Lai and Wei (2005). The coupling is induced through the inclusion of the $x_{1}$ term in the equation of $\dot{x}_{2}$ with coupling strength $\epsilon$. Related systems have been discussed before in Guan, Lai and Wei (2005), Quian Quiroga, Kreuz and Grassberger (2002a), Paluš et al. (2001), Paluš and Vejmelka (2007). In our study, coupling strengths $\epsilon$ between 8.0 and 12.0 are used (see below).

The solution is approximated by integration from 0 to 50 with step size 0.01 by using a fourth-order Runge-Kutta algorithm (cf. Press et al., 1992) leading to 5000 data points. Note that the above notation is common in physics but in a mathematical sense not correct (there are derivatives on the left-hand side and discrete time noise on the right-hand side). The precise meaning is, that in the discrete approximation with the Runge-Kutta algorithm the right-hand side is used at each gridpoint in time with additional discrete noise. To make the setting more realistic, stationary observation noise is added to the oscillators: To the computed values $x_{i, t}, y_{i, t}, z_{i, t}$ at time $t$, we add $u_{t}^{i}=0.9 u_{t-1}^{i}+v_{t}^{i}$ with $v_{t}^{i} \sim \mathcal{N}(0,0.1)$.

The trajectory of the Lorenz attractor (in the $x z$ plane) for $\epsilon=0$ (no coupling) and $\epsilon=12$ (coupling) are given in Figure 1. It can be observed that, in contrast to the Rössler attractor [see Figure 2(a)-(c)], the Lorenz attractor has two rotation centers in the uncoupled case. Therefore, the phase of the Lorenz attractor is usually defined in the $u z$-plane where $u=\sqrt{x^{2}+y^{2}}$ (cf. Pikovsky et al., 1997). In the $u z$-plane, only one rotation center exists which is illustrated in Figure 2(d)(f).

1. Determination of the phases: We apply the Hilbert transform to estimate the phases as described in Section 2.1. The Hilbert transform is applied to the $x$ coordinate of the Rössler attractor and to the $u$ coordinate of the Lorenz attractor, using a rolling window of 1000 data points. After phase estimation, we have removed 

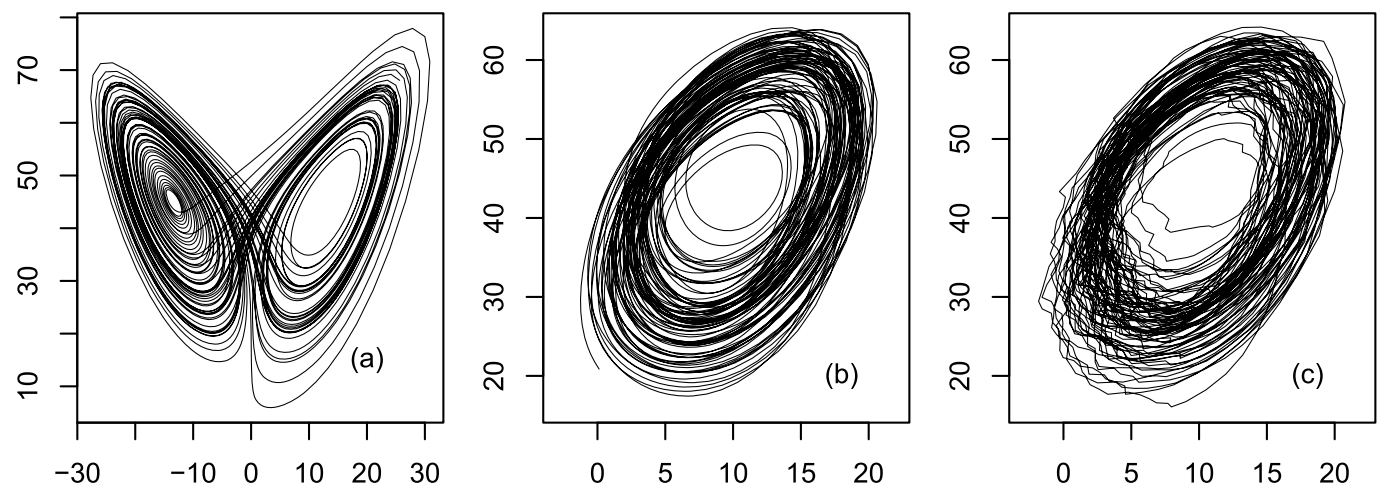

FIG. 1. Rössler-Lorenz system: Trajectory of the Lorenz attractor in the xz-plane $\left(z_{2}\right.$ vs. $\left.x_{2}\right)$ for coupling strengths $\epsilon=0$ (a) and $\epsilon=12$ (b). (c) shows the trajectory in (b) corrupted by noise.

the first and last 500 data points finally leading to 4000 data points.

Figure 3(a), (b) show 500 data points of the $x$ and $u$ coordinate of the Rössler and Lorenz attractors, respectively, for the uncoupled case $(\epsilon=0)$ and the coupled case $(\epsilon=12)$. In (c), (d), the wrapped phase estimates are given. It can be seen that in the uncoupled case the mean frequency of the Rössler system is smaller than the mean frequency of the Lorenz system.
In fact, for the given configuration the natural frequencies of the Rössler system and the Lorenz system are $\omega_{R}=0.129$ and $\omega_{L}=0.137$, respectively (see below), that is, the natural frequencies differ significantly in the uncoupled system. In (e), (f), the unwrapped phases are plotted and in $(\mathrm{g})$, (h) the "stochastic trend" (in terms of cointegration), that is, the unwrapped phases minus $0.129 t$ are plotted (the latter being the deterministic trend of the Rössler-attractor and the coupled system as
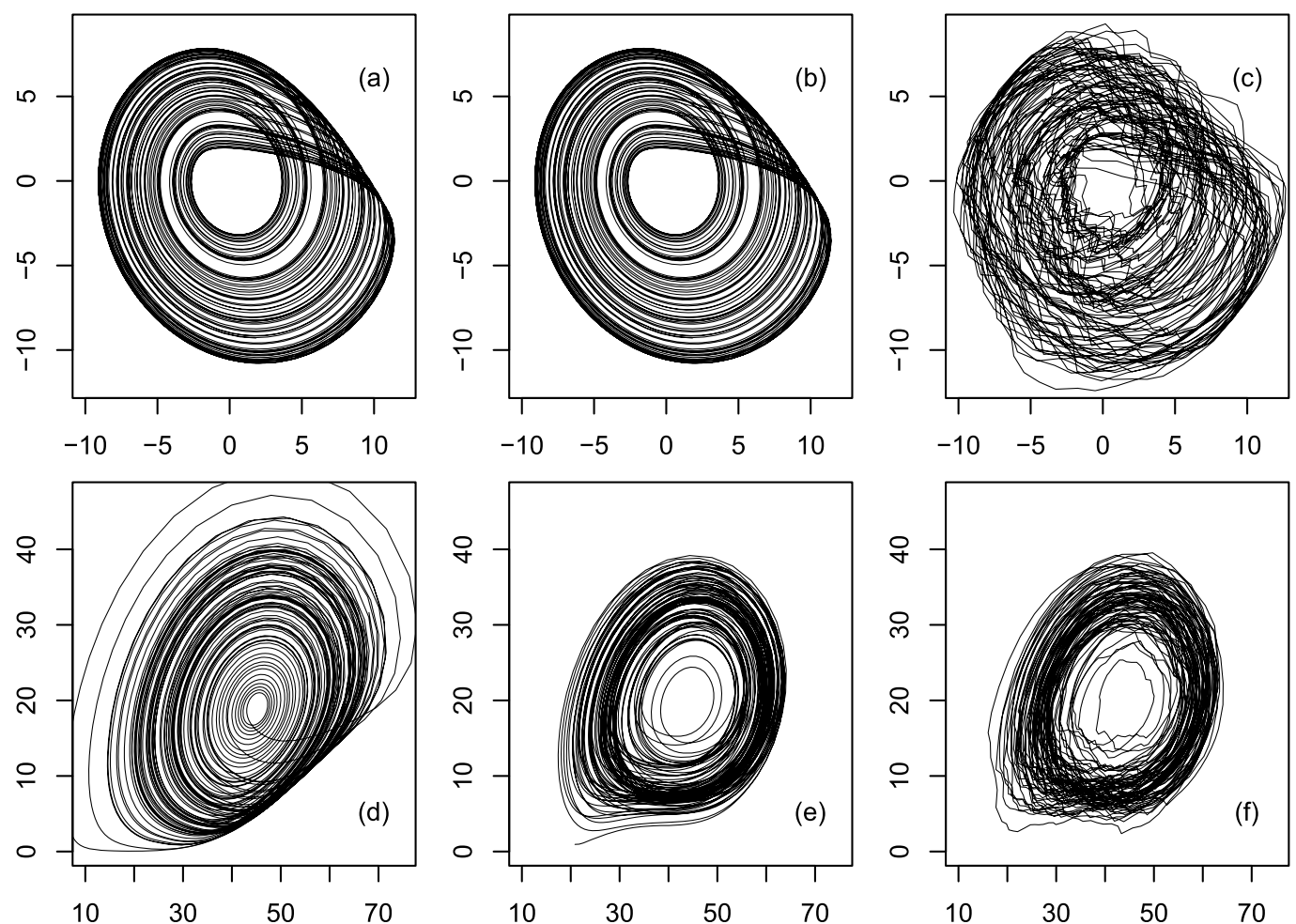

FIG. 2. Rössler-Lorenz system: Trajectories of the Rössler attractor in the xy-plane (a)-(c) ( $y_{1}$ vs. $\left.x_{1}\right)$ and Lorenz attractor in the uz-plane (d)-(f) $\left(z_{2}\right.$ vs. $\left.u_{2}\right)$ for coupling strengths $\epsilon=0$ (a), (d) and $\epsilon=12$ (b), (e) [as a result of the unidirectional coupling (a) and (b) are identical]. (c)/(f) show the trajectories in (b)/(e) corrupted by noise. 

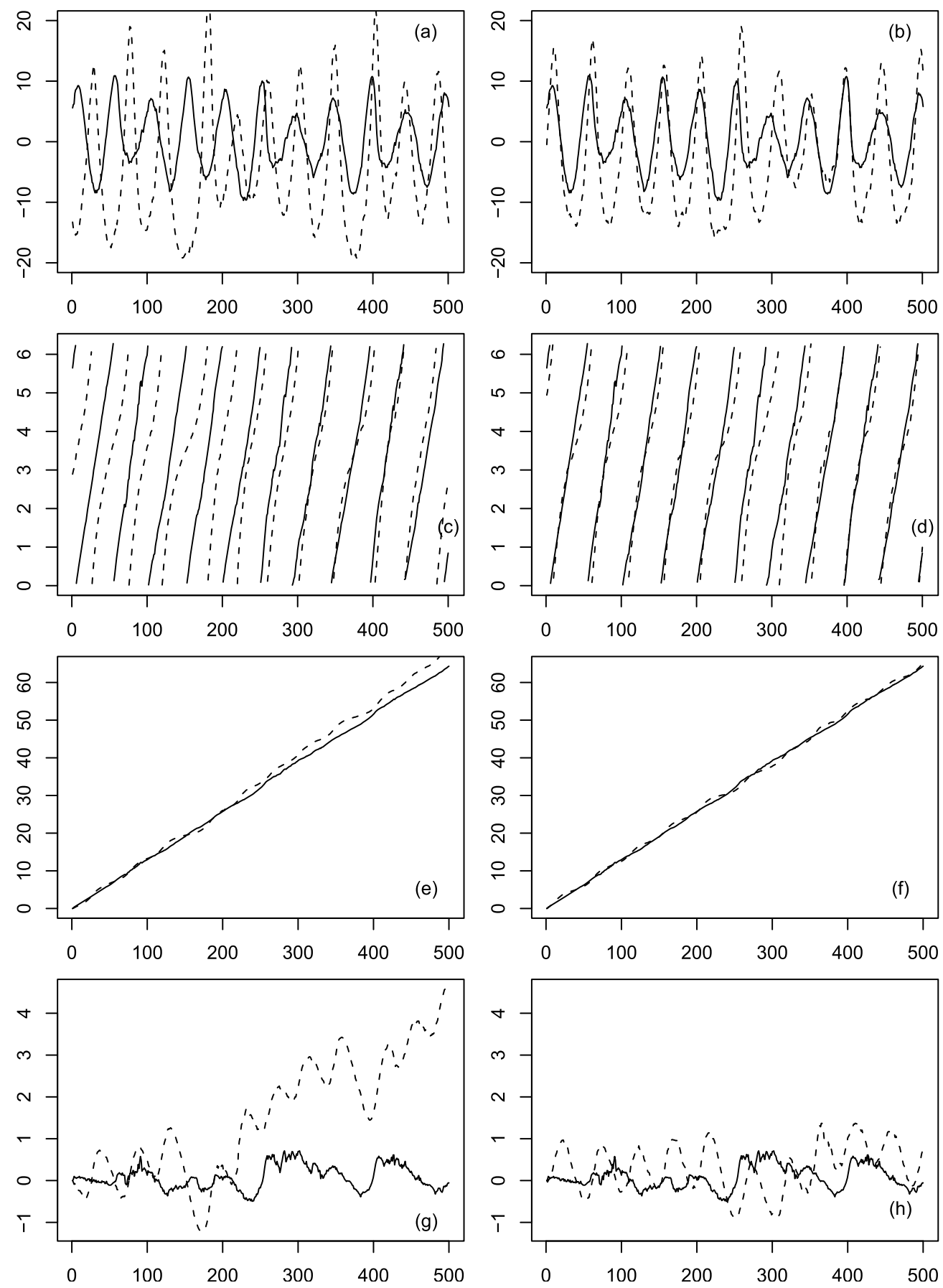

FIG. 3. Rössler-Lorenz system: 500 data points of the x coordinate of the Rössler attractor (solid lines) and of the u coordinate of Lorenz attractor (dashed lines) for $\epsilon=0$ (a) and $\epsilon=12$ (b). The data points are corrupted by noise. (c), (d) give the corresponding wrapped phase estimates, (e), (f) the unwrapped phases, and (g), (h) the stochastic trend of the unwrapped phases. 
determined below). The shape of the stochastic trend in (g) already indicates no cointegration (phase synchronization) while in (h) it indicates cointegration. The oscillation of the phase of the Lorenz attractor is due to the fact that the Lorenz attractor has a slightly steeper ascent than a descent (this can be overcome by choosing a higher AR-model order; see the comments in Appendix B).

2. and 2a. Testing for phase synchronization: We first apply the Johansen rank test in the "top $\rightarrow$ bottom'-version where we set the AR-order for simplicity to $p=2$. For example, for $\epsilon=12$ the hypothesis $\mathcal{H}(0)$ is clearly rejected with a LR-value of 92.44 (5\%-critical value: 15.41 ) and the hypothesis $\mathcal{H}(1)$ is clearly not rejected (i.e., accepted) with a LR-value of 0.01 (5\%-critical value: 3.84 ). Thus the test reveals rank $\Pi=1$, and we conclude to phase synchronization. Furthermore, the procedure also detects the $1: 1$ relation of the phase synchronization from the corresponding eigenvector.

Figure 4(a) shows the values of the $\mathcal{H}(0)$-test statistic for different $\epsilon$ (but the same values for the noise variates). The dashed line and the dotted line are the critical values 15.41 and 19.62 for the significance levels 0.05 and 0.01 , respectively (cf. Juselius, 2006, Appendix A, Case 3). For those $\epsilon$ where the test rejects the hypothesis $\mathcal{H}(1)$ was tested afterward and in all cases not rejected. Thus the plotted value of the $\mathcal{H}(0)$-statistic determined the decision phase synchronization versus no phase synchronization. The values for $\epsilon>11.1$ are all highly significant corresponding to phase synchronization while the values for $\epsilon \leq 11.1$ are nearly all not significant indicating no phase synchronization.

In Figure 4(c), the phase synchronization index $\hat{R}^{2}$ is plotted. The associated critical values are computed as explained in Schelter et al. (2007). The results of the two tests agree apart from $\epsilon=9.6,10.2,11.0,11.1$ (and 10.6 as a "borderline"-case). The difference can in all 4 cases be explained by $2 \pi$-jumps of the phasedifferences which are penalized by the LR-test but not penalized by the $\hat{R}^{2}$-statistic. The situation is highlighted in Figure 5 where we have plotted the estimated phase differences $\phi_{t}^{(1)}-\phi_{t}^{(2)}$ for $\epsilon=0,9.6,10.2$ and 12 (the situation for $11.0,11.1$ being similar to the cases 9.6 and 10.2). We think it is a matter of taste whether one calls the cases $9.6,10.2$ phase synchronized (see the comments below).

We have also used the testing procedure as described under 2a based on the ADF-test. Here, we have to specify the possible phase synchronization relation in ad- vance. Based on the plot in Figure 3(b), we have decided to test for a $1: 1$ synchronization relation, that is we have applied the ADF-test to $\phi_{t}^{(1)}-\phi_{t}^{(2)}$. The results are given in Figure 4(b) with the vertical axis being upside down. The dashed line and the dotted line are the critical values -1.65 and -2.33 associated with the significance levels 0.05 and 0.01 , respectively (from the standard normal distribution; see Hamilton, 1994, page 529, Case 3). The results coincide with the LR-test (with a slight difference for $\epsilon=10.5,10.6,10.7)$.

Figure 3(d)-(f) show the median and the $10 \%$ and $90 \%$ quantiles of a larger number of simulations where the cases with phase jumps have been removed. The plots and the severe limitations of this simulation are discussed in detail in the last paragraph of this section. As a positive outcome the plots confirm that the tests behave similarly. They also indicate that the ADF-test has a higher power than the Johansen rank test for values of $\epsilon$ between 9.5 and 10.5 which is not surprising since the ADF-test uses as an additional information the form of the cointegration relation (however, the true power of the tests is unknown and cannot be estimated from this simulation).

It is remarkable that the LR-test and the ADF-test as two standard tests from the theory of cointegration perform similar to the $\hat{R}^{2}$-test which has been explicitly tailored for phase synchronization. The tests are only different for processes with phase jumps which we regard more as a feature of chaotic oscillators than of real data; see also the discussion in Section 6. We also mention that we do not regard the phase synchronization index $\hat{R}^{2}$ from Figure 4(c) as some "gold-standard." Instead we think that, for example, a (properly adjusted) LR-test in an adequate model would be a better choice.

3. and 3a. Estimation, further testing and unidirectional coupling: Given that $\phi_{t}^{(1)}, \phi_{t}^{(2)}$ are cointegrated with cointegrating vector $\beta=(1,-1)^{\prime}$, we estimate the VEC-model (15), (16) to analyze the joint dynamics with the aim to uncover the directional coupling. Here we restrict to the case $\epsilon=12$ from Figure 3(a)-(c). The estimation results are given in Table 1.

In the equation of $\Delta \phi_{t}^{(1)}$ only $\mu_{1}$ and $\gamma_{1}$ have a significant t-ratio. In the equation of $\Delta \phi_{t}^{(2)}$ all parameters except $\delta_{2}$ are highly significant. Since $\delta_{1}$ and $\delta_{2}$ as well as the cross-correlations of the residuals with $\operatorname{Corr}\left(\eta_{t}^{(1)}, \eta_{t}^{(2)}\right)=0.022$ are not significantly different from zero, the dependency of the phases is explained in the model purely by the error correction mechanism. Since $\alpha_{1}=0$ and $\alpha_{2}>0$, the VEC-model reliably identifies the direction of coupling of the original Rössler-Lorenz system. 

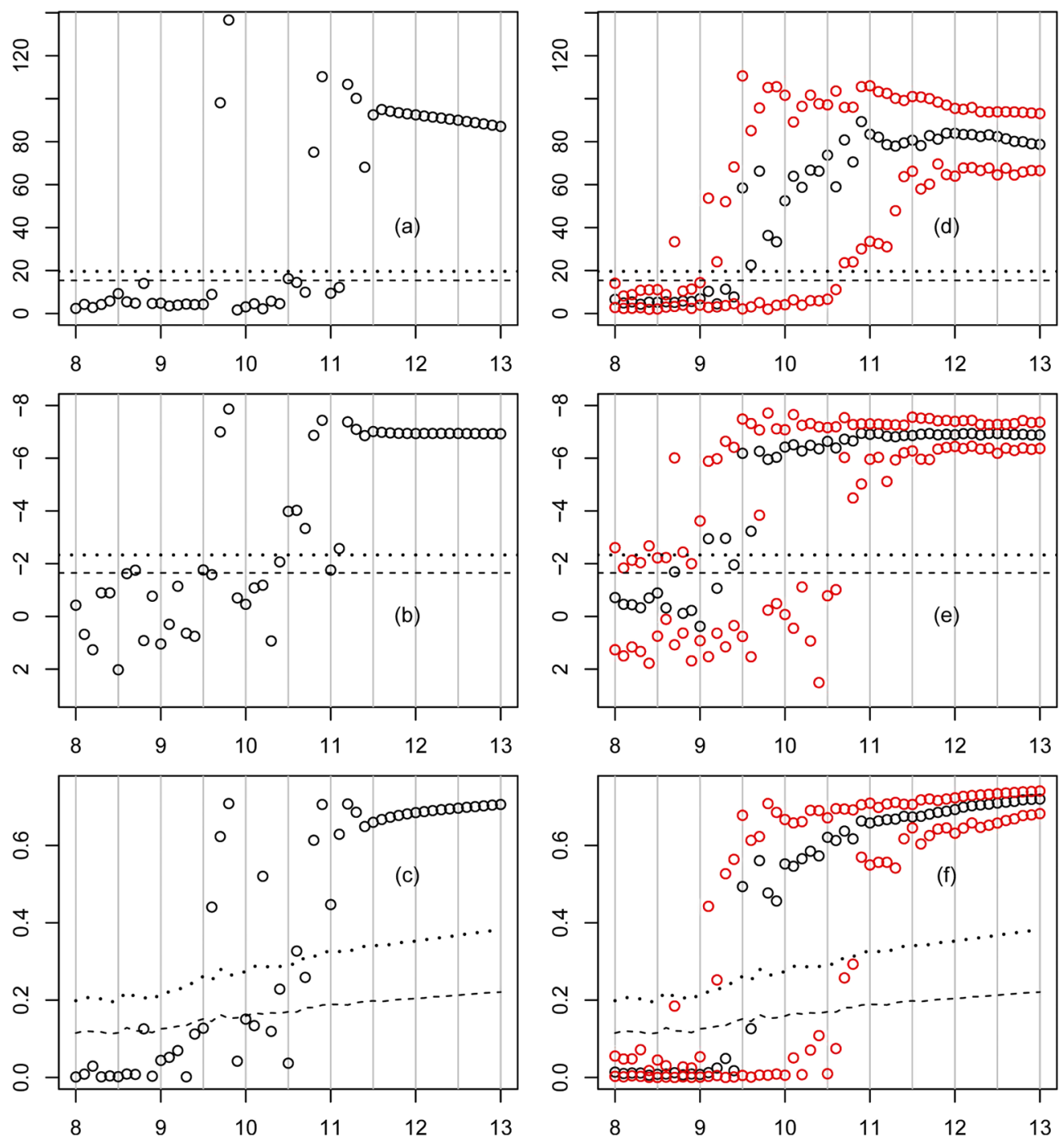

FIG. 4. Rössler-Lorenz system: (a), (d) values of the LR-test for $\mathcal{H}(0)$; (b), (e) values of the ADF-test; (c), (f) values of the phase synchronization index $\hat{R}^{2}$. (a)-(c) show the results of one simulation for each coupling strength $\epsilon$ between 8 and 13; (d)-(f) show the median and the $10 \%$ and $90 \%$ quantiles of a larger simulation study. The dashed lines and the dotted lines show the significance levels $\alpha=0.05$ and $\alpha=0.01$, respectively.

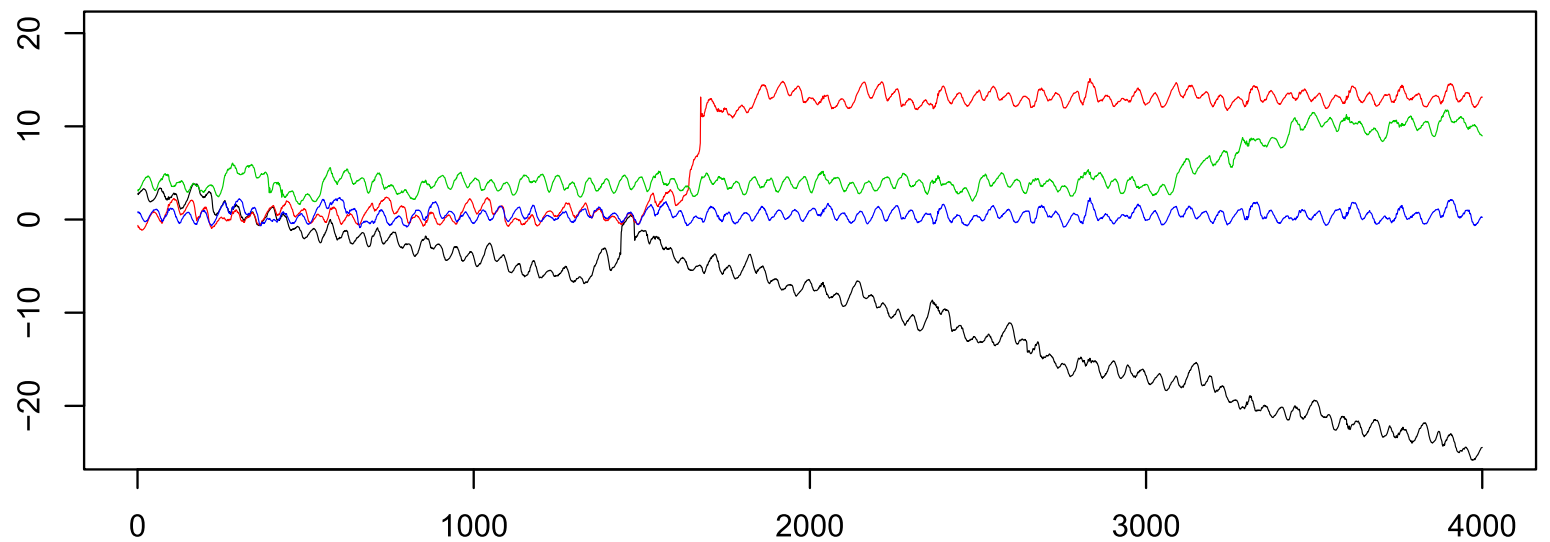

FIG. 5. Rössler-Lorenz system: Phase differences $\phi_{t}^{(1)}-\phi_{t}^{(2)}$ for coupling strength $\epsilon=0$ (black), $\epsilon=9.6($ green $), \epsilon=10.2($ red $)$ and $\epsilon=12$ (blue). 
TABLE 1

Estimated VEC-model for the Rössler-Lorenz system

\begin{tabular}{lllrc}
\hline Parameter & Estimate & Std. error & t-ratio & p-value \\
\hline$\alpha_{1}$ & 0.0009 & 0.0015 & 0.62 & 0.53 \\
$\gamma_{1}$ & 0.059 & 0.016 & 3.71 & 0.0002 \\
$\delta_{1}$ & 0.024 & 0.014 & 1.70 & 0.089 \\
$\mu_{1}$ & 0.1177 & 0.0029 & 41.12 & $<0.0001$ \\
$\alpha_{2}$ & 0.0110 & 0.0011 & 9.65 & $<0.0001$ \\
$\gamma_{2}$ & 0.726 & 0.011 & 68.38 & $<0.0001$ \\
$\delta_{2}$ & 0.028 & 0.012 & 2.38 & 0.017 \\
$\mu_{2}$ & 0.0262 & 0.0022 & 12.11 & $<0.0001$ \\
\hline
\end{tabular}

Finally, a reestimation of the model with only the significant parameters yields

$$
\begin{aligned}
\Delta \phi_{t}^{(1)}= & 0.060 \Delta \phi_{t-1}^{(1)} \\
& +0.121+\eta_{t}^{(1)} \\
\Delta \phi_{t}^{(2)}= & 0.011\left(\phi_{t-1}^{(1)}-\phi_{t-1}^{(2)}\right) \\
& +0.727 \Delta \phi_{t-1}^{(2)}+0.030+\eta_{t}^{(2)}
\end{aligned}
$$

Note also the more informative representation (24) below and its discussion.

We now discuss the estimated system in terms of the modified VEC-representation (14) and the MArepresentation (11). At the same time, this system is a nice illustration of the theoretical setting presented in Section 3.1 (and a nice example for a cointegrated system). With the above estimates, we obtain

$$
\begin{aligned}
& \beta=\left(\begin{array}{c}
1 \\
-1
\end{array}\right), \quad \beta_{\perp}=\left(\begin{array}{l}
1 \\
1
\end{array}\right), \\
& \alpha=\left(\begin{array}{c}
0 \\
0.011
\end{array}\right), \quad \alpha_{\perp}=\left(\begin{array}{l}
1 \\
0
\end{array}\right), \quad \mu=\left(\begin{array}{l}
0.121 \\
0.030
\end{array}\right) .
\end{aligned}
$$

This leads with straightforward calculation to the other values

$$
\begin{gathered}
\Gamma_{1}=\left(\begin{array}{cc}
0.06 & 0 \\
0 & 0.73
\end{array}\right), \\
\Gamma=\left(\begin{array}{cc}
0.94 & 0 \\
0 & 0.27
\end{array}\right), \\
C=0.94^{-1}\left(\begin{array}{l}
1 \\
1
\end{array}\right)(1,0), \\
\omega_{0}=C \mu=0.129\left(\begin{array}{l}
1 \\
1
\end{array}\right),
\end{gathered}
$$

and $\beta_{0}=0.49$. With these values, we obtain the VECrepresentation

$$
\begin{aligned}
& {\left[\left(\begin{array}{c}
\Delta \phi_{t}^{(1)} \\
\Delta \phi_{t}^{(2)}
\end{array}\right)-\left(\begin{array}{l}
0.129 \\
0.129
\end{array}\right)\right]} \\
& =\left(\begin{array}{c}
0 \\
0.011
\end{array}\right)\left(\phi_{t-1}^{(1)}-\phi_{t-1}^{(2)}-0.49\right) \\
& \quad+\left(\begin{array}{cc}
0.06 & 0 \\
0 & 0.73
\end{array}\right)\left[\left(\begin{array}{c}
\Delta \phi_{t-1}^{(1)} \\
\Delta \phi_{t-1}^{(2)}
\end{array}\right)\right. \\
& \left.\quad-\left(\begin{array}{c}
0.129 \\
0.129
\end{array}\right)\right]+\eta_{t}
\end{aligned}
$$

and the MA-representation

$$
\begin{aligned}
\phi_{t}= & \left(\begin{array}{l}
1 \\
1
\end{array}\right) \sum_{i=1}^{t} \eta_{i}^{(1)}+\left(\begin{array}{l}
0.129 \\
0.129
\end{array}\right) t \\
& +C^{*}(L)\left(\eta_{t}+\mu\right)+\phi_{0} .
\end{aligned}
$$

Equation (24) has the meaning that the phase process is pulled toward the equilibrium defined by $\beta^{\prime} \phi_{t}-\beta_{0}=$ $\phi_{t}^{(1)}-\phi_{t}^{(2)}-0.49=0$ with the force $\alpha=\left(\begin{array}{c}0 \\ 0.011\end{array}\right)$ which gets active as soon as the process leaves the equilibrium. In this case, $\alpha_{1}=0$, that is, the correction is on process 2 only. This means that the unidirectional coupling between the Rössler and the Lorenz system has been detected correctly.

The equilibrium $\phi_{t}^{(1)}-\phi_{t}^{(2)}-0.49=0$ means that the phase of the Rössler system is ahead of the phase of the Lorenz system in the average by 0.49 . This is confirmed by the plot in Figure 3(d).

The representation (20) has the meaning that both phases are mainly pushed in direction $\left(\begin{array}{l}1 \\ 1\end{array}\right)$ — both with a deterministic and a random walk component. The stationary component $C^{*}(L)\left(\eta_{t}+\mu\right)$ (whose precise form is complicated) acts as a disturbance component pushing the system with little shocks again and again out of the equilibrium.

For comparison, we also fit the phase model $\Delta \phi_{t}=$ $\gamma \Delta \phi_{t-1}+\mu+\eta_{t}$ to each oscillator in the uncoupled case $\epsilon=0$ which gives

$$
\begin{aligned}
& \left(\Delta \phi_{t}^{(1)}-0.129\right) \\
& \quad=0.060\left(\Delta \phi_{t-1}^{(1)}-0.129\right)+\eta_{t}^{(1)}, \\
& \left(\Delta \phi_{t}^{(2)}-0.137\right) \\
& \quad=0.735\left(\Delta \phi_{t-1}^{(2)}-0.137\right)+\eta_{t}^{(2)},
\end{aligned}
$$

[(17) and (21) are of course identical]. As mentioned above the natural frequencies of the Rössler and the 
Lorenz system therefore are $\omega_{R}=0.129$ and $\omega_{L}=$ 0.137 , respectively, that is, the natural frequencies differ significantly in the uncoupled system, while in the coupled system they both are $\omega=0.129$ (remember that $\omega t$ is the deterministic part of the trend).

One effect should be kept in mind: The prior application of the Hilbert transform leads to phases which are smoother than the original "true" ones. This effect needs to be investigated in the future (see Appendix A). As a consequence, the estimated variances of $\eta_{t}$ and $\phi_{t}$ will usually be smaller than the true ones. In (21), (22) one finds $\operatorname{Var} \eta_{t}^{(1)}=0.0034$ and $\operatorname{Var} \eta_{t}^{(2)}=0.0025$ leading to $\operatorname{Var}\left(\Delta \phi_{t}^{(1)}\right)=0.0034$ and $\operatorname{Var}\left(\Delta \phi_{t}^{(2)}\right)=0.0054$. This means that the Lorenz attractor has a more varying frequency than the Rössler attractor and it is likely that this also holds for the true frequencies.

Perhaps the most important aspect of this fitted VECmodel is that the direction of coupling is detected correctly. In Figure 3(d), in total 1203 series have been tested positively as being phase-synchronized with the LR-test. Out of these, $93.6 \%$ have been tested by the VEC-model as having unidirectional coupling. This decision was characterized by a p-value larger than 0.1 for $\alpha_{1}$ and a p-value smaller than 0.01 for $\alpha_{2}$. This means that in more than $93.6 \%$ cases of the synchronized one sided-coupled Rössler-Lorenz system a testing value smaller than the $90 \%$-quantile of the stochastic cointegration-model was obtained for $\alpha_{1}$. This also confirms that the stochastic model is reasonable.

We finally mention the limitations of sampling from the Rössler-Lorenz system, and of the plots in Figure 3(d)-(f) for the purpose of this paper: As a chaotic oscillator the Rössler-Lorenz system is very sensitive to the starting values and also to the random inputs $w_{1}$ and $w_{2}$ leading under the simulation roughly to 4 different path-types: synchronized oscillators, nonsynchronized oscillators without phase jumps (over the whole segment), oscillators with phase jumps, segmentwise combinations of the former three cases. Even for the fist two cases the approximation with the cointegration model of this paper leads to very different parameter values and, therefore, to different models from a stochastic viewpoint. For this reason, simulations from the Rössler-Lorenz system are not suitable, for example, judging the properties of estimates or tests in detail (with the above remarks on the higher power of the ADF-test and the suitability for detecting unidirectional coupling as an exception). In detail for Figure 3(d)-(f), we have done 100 simulations for each $\epsilon$ (with the same noise variates). Out of a total of 5100 paths, those paths with phase jumps and segmentwise changing structure have been removed by ad hoc testing and eye-inspection, leading to only 1530 cases (e.g., $\epsilon=8.8,9.2,9.4,9.6,9.7$ less than 14 values remained) which is the reason for the erratic behavior of the quantiles in Figure 3(d)-(f). Of course, the subjective method of excluding the other cases is questionable and it makes no sense to repeat the procedure for a larger number of simulations. To summarize the cointegration model of this paper is not a statistical model for the Rössler-Lorenz system as a whole but only for single paths of it, and moreover, for the detection of phase synchronization of other time series not stemming from chaotic oscillators.

\subsection{Synchronization of Electrochemical Oscillators}

We now investigate with the above methods phase synchronization in a chaotic process in electrochemistry. The system is the electrodissolution of nickel in sulfuric acid. The electrodissolution takes place on two nickel wires, and the rate of the dissolution (corrosion rate), measured as the current, exhibits chaotic behavior; see Kiss and Hudson (2002). The oscillators are coupled through a set of serial and parallel resistors. Such coupling induces a bidirectional, electrical interaction between the electrochemical processes. For more details and a schematic diagram of the experimental apparatus, see Kiss and Hudson (2002).

The phase synchronization of the chaotic chemical process was established using nonstatistical methods (an entropy based measure of the cyclic phase difference distribution) in Kiss and Hudson (2002) and Kiss, Lv and Hudson (2005). In this section, we explore the characteristics of the synchronization process using the cointegration technique. The Hilbert transform is applied to estimate the phases of the two-dimensional time series, and afterward more than 5 seconds of data points are removed at the beginning and the end. A data set is considered with data acquisition rate of $25 \mathrm{~Hz}$ leading to about 21 data points per cycle. The whole series then consists of 37502 -dimensional data points. In order to test the methods on a smaller data set, we split this in 6 segments of equal length each consisting of 625 data points. The results on each of the 6 segments are essentially the same and for clarity we therefore report only the results for one segment (instead of displaying summary statistics etc.). Furthermore, we mention some results for the whole stretch of 3750 data points. Figure 6 shows 300 data points of the two oscillators from the investigated segment (the first 
component is in blue, the second in red), the estimated phases before unwrapping and the phase differences $\Delta \phi_{t}$. The plot reveals that the phases and the phasedifferences have an in-cycle fluctuation which comes from the phase slowing down at the minimum of the oscillations and speeding up at the maximum.

The AR-order $p$ in (10) is chosen by inspection of the significant lags in the model fit below leading to the choice $p=6$. This order is necessary because of the above mentioned in-cycle fluctuations. The red peak for the phase differences is due to the fact that the corresponding cycle is rather short resulting in large phase differences. As above, the Johansen LR test and the ADF test are applied to test for cointegration of the phases and therefore for phase synchronization of the original series. The hypothesis $\mathcal{H}(0)$ is clearly rejected with a LR-value of 52.68 (5\%-critical value: 17.95$)$ and the hypothesis $\mathcal{H}(1)$ is clearly not rejected (i.e., accepted) with a LR-value of 0.02 (5\%-critical value: 8.18 ). Thus the test reveals $\operatorname{rank} \Pi=1$, and we conclude to phase synchronization. Furthermore, the procedure also detects the $1: 1$-relation of the phase synchronization from the corresponding eigenvector. For the long series consisting of 3750 data the LR-value for $\mathcal{H}(0)$ is even 321.94 and for $\mathcal{H}(1)$ it is 0.03 . The ADFtest leads with a testing value of -6.398 and a $1 \%$ critical value of -3.43 to the rejection of the hypothesis that the phase are not cointegrated. The same result is obtained with a testing value of -17.9464 for the long series, that is, the ADF-test also confirms phase synchronization. Given that $\phi_{t}^{(1)}, \phi_{t}^{(2)}$ are cointegrated with cointegrating vector $\beta=(1,-1)^{\prime}$ we estimate the VEC-model (10) with order $p=6$ to analyze in more detail the joint dynamics. Different to the results in Section 5.1 several AR-parameters are significant. The estimated coefficients are displayed in Table 2.

As expected, there is no unidirectional coupling which is reflected by $\alpha_{1}<0$ and $\alpha_{2}>0$. It is however remarkable that $\alpha_{2}$ is 3-times as large as $\alpha_{1}$. On the whole segment with 3750 data, we obtain as estimates $\alpha_{1}=-0.012$ and $\alpha_{2}=0.026$, that is, in total $\alpha_{2}$ is more than twice as large as $\alpha_{1}$. The same holds on all 6 segments and also when trying different ARorders. In addition, the AR-coefficients $\Gamma_{\mathrm{k}}$ indicate an influence from series 2 to series 1 . Summarizing, the force toward the phase equilibrium is much stronger from series 2 on series 1 than vice versa. These results thus show that while the physical form of the coupling is symmetrical (through differences in the electrode potentials, Kiss and Hudson, 2002), the effective
TABLE 2

Estimated VEC-model for the electrochemical oscillators. Note that all other coefficients $\Gamma_{1_{i j}}, \ldots, \Gamma_{5_{i j}}$ are not significantly different from 0

\begin{tabular}{lrrrr}
\hline Parameter & Estimate & Std. error & t-ratio & p-value \\
\hline$\alpha_{1}$ & -0.011 & 0.003 & -3.72 & 0.0002 \\
$\mu_{1}$ & 0.073 & 0.008 & 8.96 & $<0.0001$ \\
$\Gamma_{1_{11}}$ & 1.565 & 0.040 & 38.65 & $<0.0001$ \\
$\Gamma_{2}$ & -1.150 & 0.075 & -15.36 & $<0.0001$ \\
$\Gamma_{31}$ & 0.567 & 0.086 & 6.61 & $<0.0001$ \\
$\Gamma_{41}$ & -0.206 & 0.076 & -2.71 & 0.007 \\
$\alpha_{2}$ & 0.033 & 0.006 & 5.62 & $<0.0001$ \\
$\mu_{2}$ & 0.102 & 0.017 & 5.97 & $<0.0001$ \\
$\Gamma_{1}$ & 0.427 & 0.084 & 5.06 & $<0.0001$ \\
$\Gamma_{12}$ & 0.740 & 0.040 & 18.57 & $<0.0001$ \\
$\Gamma_{22}$ & -0.444 & 0.156 & -2.84 & 0.005 \\
$\Gamma_{22}$ & -0.142 & 0.050 & -2.85 & 0.005 \\
$\Gamma_{5}$ & -0.207 & 0.088 & -2.35 & 0.019 \\
\hline
\end{tabular}

coupling on the phase dynamics could develop asymmetries, likely due to the heterogeneities in the local dynamics.

We finally discuss the estimated system in terms of the modified VEC-representation (14). The above table yields

$$
\begin{aligned}
& \beta=\left(\begin{array}{c}
1 \\
-1
\end{array}\right), \quad \beta_{\perp}=\left(\begin{array}{l}
1 \\
1
\end{array}\right), \\
& \alpha=\left(\begin{array}{c}
-0.011 \\
0.033
\end{array}\right), \quad \alpha_{\perp}=\left(\begin{array}{l}
0.033 \\
0.011
\end{array}\right), \quad \mu=\left(\begin{array}{l}
0.073 \\
0.102
\end{array}\right)
\end{aligned}
$$

plus the values for the $\Gamma_{k}$ (not listed here) leading with $\Gamma=I_{p}-\sum_{i=1}^{p-1} \Gamma_{i}, C=\beta_{\perp}\left[\alpha_{\perp}^{\prime} \Gamma \beta_{\perp}\right]^{-1} \alpha_{\perp}^{\prime}, \bar{\alpha}=$ $\alpha\left(\alpha^{\prime} \alpha\right)^{-1}$ to

$$
\omega_{0}=C \mu=0.302\left(\begin{array}{l}
1 \\
1
\end{array}\right)
$$

and

$$
\beta_{0}=\bar{\alpha}^{\prime}\left[\Gamma C-I_{d}\right] \mu=0.077
$$

meaning that the "true" estimated equilibrium is $\phi_{t}^{(1)}$ $\phi_{t}^{(2)}-0.077=0$. This leads to the VEC-representation

$$
\begin{gathered}
{\left[\left(\begin{array}{c}
\Delta \phi_{t}^{(1)} \\
\Delta \phi_{t}^{(2)}
\end{array}\right)-\left(\begin{array}{c}
0.302 \\
0.302
\end{array}\right)\right]} \\
=\left(\begin{array}{c}
-0.011 \\
0.033
\end{array}\right)\left(\phi_{t-1}^{(1)}-\phi_{t-1}^{(2)}-0.077\right) \\
\quad+\text { higher order AR-terms }+\eta_{t}
\end{gathered}
$$

which demonstrates the error correcting effect on the first and second component if the oscillators are out 

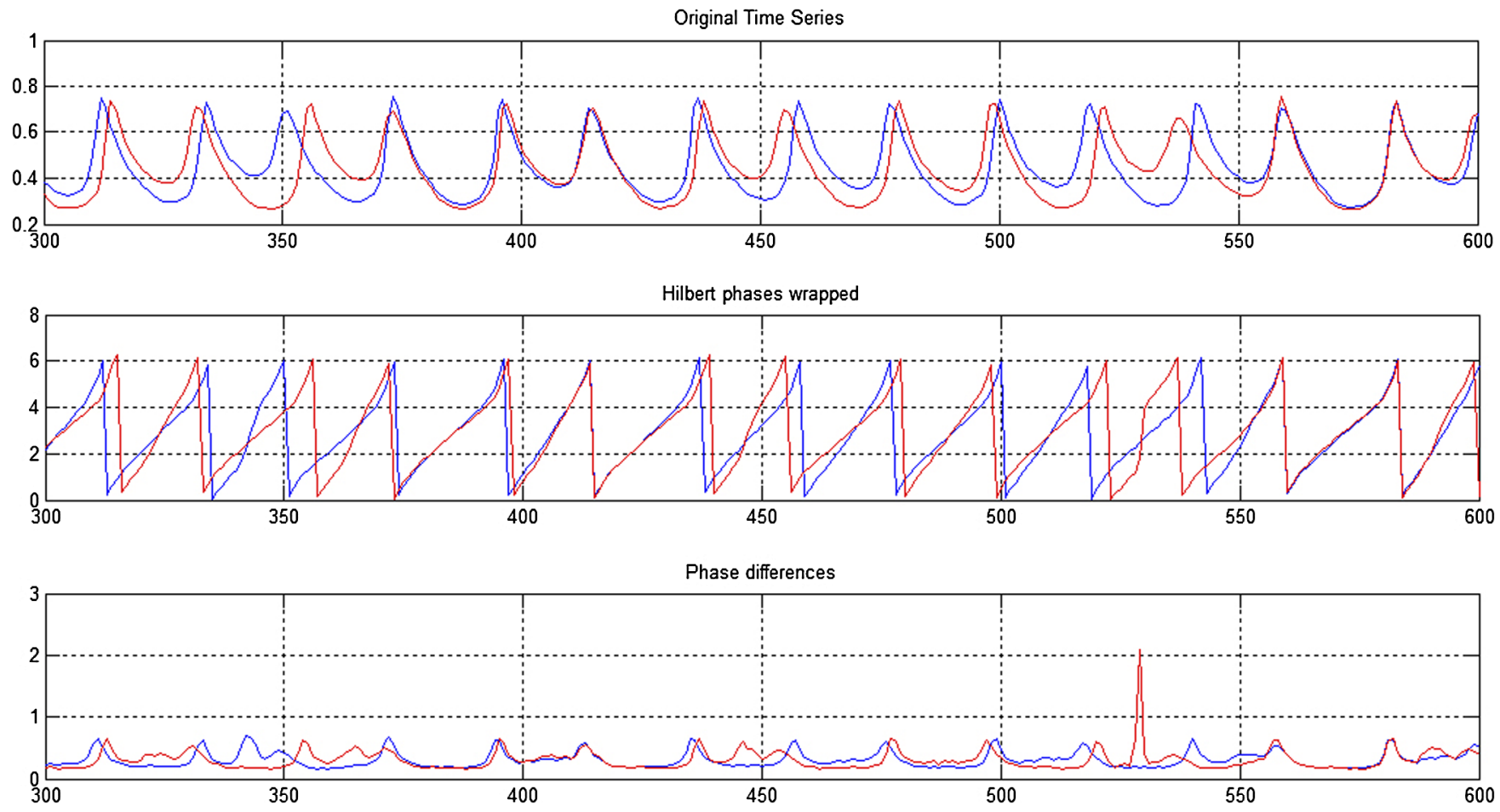

FIG. 6. Currents at 2 electrodes of an electrochemical oscillator: The figures show the original currents, the Hilbert estimate of the phases before unwrapping, and the phase differences $\Delta \phi_{t}$ for each phase process (the first component is in blue, the second in red).

of equilibrium. For completeness, we mention that the corresponding fit to the long series leads to

$$
\begin{aligned}
& {\left[\left(\begin{array}{c}
\Delta \phi_{t}^{(1)} \\
\Delta \phi_{t}^{(2)}
\end{array}\right)-\left(\begin{array}{c}
0.302 \\
0.302
\end{array}\right)\right]} \\
& =\left(\begin{array}{c}
-0.012 \\
0.026
\end{array}\right)\left(\phi_{t-1}^{(1)}-\phi_{t-1}^{(2)}-0.152\right) \\
& \quad+\text { higher order AR-terms }+\eta_{t} .
\end{aligned}
$$

In total, the methods from cointegration work well with respect to phase synchronization on the dataset. One critical issue is that the phases differences in the lower plot of Figure 6 can hardly be viewed as Gaussian random variables. A density plot shows clearly a skewed distribution for the phase differences which potentially leads to different p-values of the test statistics. To investigate this, we have simulated the distribution of the error-correction coefficients $\alpha_{i}$ under the hypothesis $\alpha_{i}=0$ in this example with a parametric VAR-bootstrap. The results surprisingly indicate that the Gaussian approximation is reasonable. Details are omitted since this topic needs a deeper investigation which is postponed to future work.

\section{CONCLUSION AND OUTLOOK}

We have pointed out the connection between the theory of cointegration and the theory of phase synchronization. In particular, a cointegrated dynamical system can be used as a stochastic model for a multivariate phase process which describes the behavior of the phases in detail. Contrary to other concepts like the spectral coherence which only describe the properties of the phase processes, we now have a specific model in the time domain for the dynamics of the phase processes. The model leads to the characterization and identification of the equilibrium relations related to phase synchronization: For example, the unknown coupling structure can be revealed by using tests for unidirectional and bidirectional coupling. We have demonstrated this in applications for a coupled Rössler-Lorenz system with noise and electrochemical oscillators.

In neuroscience where phase synchronization is regarded as essential for functional coupling of different brain areas the new methods coming from cointegration may enhance the present methods like spectral analysis, correlation and coherence analysis, triplet analysis, joint phase histograms, etc. This however needs to be investigated and confirmed. 
From a physical view, the potential of cointegration for phase synchronization can perhaps best be understood from a comparison with the Kuramoto model. As pointed out in this paper, cointegration can be understood as an approximation to a stochastic Kuramototype model which can be fitted to the data (apart from the different treatment of phase shifts; see below). The cointegrated system describes both: (1) how the system is constantly pulled toward the equilibrium determined by the phase synchronization relations; and (2) how the system is constantly disturbed by little shocks and kicked out out of the equilibrium.

Another important aspect is that the cointegration model covers both: the case where the phases are synchronized and the case where they are not synchronized. As a consequence, the diagnosis on synchronization can be made from a statistical fit of the model to the data.

At the same time, the comparison to the Kuramoto model shows the difference to previous research: The Kuramoto model (6) has the equilibrium $\phi^{(j)}-\phi^{(i)} \equiv$ $0 \bmod 2 \pi \forall i, j$ while the cointegration model (7) has the equilibrium $\phi^{(j)}-\phi^{(i)}=0 \forall i, j$. This means two processes whose phase difference is shifted (quickly) by $2 \pi$ may be diagnosed as phase synchronized in the Kuramoto model while they will not be diagnosed as phase synchronized in the cointegration model. One may term this strong/weak synchronization. We advocate the view that phase jumps as in the RösslerLorenz system hardly occur with real data meaning that the cointegration model is a proper model in most cases. Nevertheless, it would be important to have similar techniques and results for the stochastic Kuramoto model. Another aspect is that phase jumps may occur erroneously caused by noise or wrong data. Of course, this raises the question of robustness of the phase estimate.

There is a wealth of possible directions for future research: An important issue is a theoretical treatment of the present model taking into account that the phases are unobserved and the present cointegration model describes the dynamics of the phases as the unobserved state in a nonlinear state space model (VEC-state oscillators; see Appendix A). It seems to be challenging to derive, for example, the asymptotic distribution of the likelihood ratio test for phase synchronization in such a setting. Another topic of interest is a larger number of oscillators. In some example (e.g., for phase synchronization of the cardiovascular and respiratory system) the correcting forces are obviously different if the system is out of the equilibrium to the positive or negative side. Here, a nonlinear cointegration system would be needed for a proper modeling.

\section{APPENDIX A: VEC-STATE OSCILLATORS}

Inspired by the motivation at the end of the introduction we define the class of

VEC-state oscillators as a general model for (possibly phase synchronized) d-dimensional oscillators with random phases. The model is the nonlinear state space model with

State equation:

$$
\begin{gathered}
\Delta \phi_{t}=\Pi \phi_{t-1}+\sum_{i=1}^{p-1} \Gamma_{i} \Delta \phi_{t-i}+\mu+\eta_{t}, \\
\eta_{t} \sim \mathcal{N}\left(0, \Sigma_{\eta}\right)
\end{gathered}
$$

(or, alternatively, a model which guarantees positivity of the phases; see below).

Observation equation:

$$
\begin{aligned}
Y_{t}^{(i)}= & a_{i} \cos \left(\phi_{t}^{(i)}\right)+b_{i}+\varepsilon_{t}^{(i)}, \\
& \varepsilon_{t} \sim \mathcal{N}\left(0, \Sigma_{\varepsilon}\right),
\end{aligned}
$$

where $\varepsilon_{t}$ and $\eta_{t}$ are mutually and serially independent. In its simplest form the amplitude $a$ and the baseline $b$ are parameter vectors. In some cases, they need to be time varying [see (ii) below]. The case $\operatorname{rank}(\Pi)=0$ is included [the case $\operatorname{rank}(\Pi)=d$ requires the additional term $v t$ in (A.1) for a meaningful phase-model; see Section 4]. If $1 \leq \operatorname{rank}(\Pi) \leq d-1$, we have phase synchronization. In that case, we prefer writing the state equation in the form

$$
\begin{aligned}
& \left(\Delta \phi_{t}-\omega_{0}\right) \\
& =\alpha\left(\beta^{\prime} \phi_{t-1}-\beta_{0}\right) \\
& \quad+\sum_{i=1}^{p-1} \Gamma_{i}\left(\Delta \phi_{t-i}-\omega_{0}\right)+\eta_{t}
\end{aligned}
$$

(see Section 3) with the phase synchronization relations $\beta^{\prime} \phi_{t}-\beta_{0}=0$, the mean phase shift $\beta_{0}$ and the deterministic trend $\omega_{0} t$. $\Pi$ is now decomposed into $\Pi=\alpha \beta^{\prime}$ with $d \times r$-matrices $\alpha$ and $\beta$ of full rank $r$.

The most common approach in physics is to estimate the phase process $\phi_{t}^{(i)}$ from the oscillator $Y_{t}^{(i)}$ by means of the Hilbert transform. In terms of state space models, one may regard this as a smoother for estimating the mean of the conditional distribution (although its properties are not clear). The approach of this paper is to use the estimated phases "as if they were observed" and to apply standard cointegration techniques to it. 
Of course, the effect of the Hilbert transform on the properties of these estimates is not clear, and the significance levels of the tests need to be investigated. This is beyond the scope of this paper, but we want to provide some heuristic arguments why everything remains the same even in this situation: Taking the conditional expectation (given $Y_{1}, \ldots, Y_{n}$ ) in (A.3) reveals that the linear structure of the conditional means is the same as before. The difference is that the variance of the innovations will be smaller (the estimated phases are smoother than the original ones) and the errors become dependent. A least squares regression based on the conditional expectations should also lead to consistent estimates for the parameters and in particular the $\mathrm{t}$-statistic for a specific parameter (which is rescaled by the empirical variance) should follow asymptotically a $\mathrm{t}$-distribution. The argument is the same for the ADFtest in the case $a \neq 0, b=0$ since this is the "regular" case where the drift dominates the stochastic trend and the asymptotic distribution of the unit root estimate is Gaussian (Fuller, 1996, Theorem 10.1.5).

For the LR-test with its nonstandard distribution, a different heuristics is needed: Inspection of the proof of the asymptotic distribution of the LR-test (Johansen, 1995, Theorem 11.1) reveals that its limiting distribution only depends on the number $d-r$ of common stochastic trends and on the model for the deterministic terms. The model for the deterministic terms is fixedso we only have to check that the number of stochastic trends remains the same for the estimated phases (estimated with the Hilbert transform) as for the original unobserved phases. To check this, we consider in this heuristics only the case of $1: 1$ phase synchronization relations, that is, where the system splits up into $d-r$ oscillators and each of the remaining $d$ oscillators is stuck to one of the former ones by the cointegration relation, that is, we have $d-r$ groups of oscillators of different size (this is a common case for phase synchronization). We now have only to check that the groups of phase processes "stay together" under the Hilbert transform. First, it is obvious that an integrated process stays integrated: The phases will constantly increase and a trend stationary process can be excluded because this is the case where the length of the cycle is almost stuck to a pregiven length and it is clear that a true phase process with variable cycles will not be transformed by the Hilbert transform into a phase process with almost constant cycle-length. In addition, it is also clear that the phase processes will stay in the same group, that is, they will not jump to another group with a different trend since this is clearly reflected by the number of cycles. Thus the number of stochastic trends should stay the same under the Hilbert transform.

We emphasize that these are only heuristic arguments. A mathematical proof that the asymptotic distributions of the test statistics stay the same would be highly welcome. It is obvious that deriving such a result may be very challenging.

A more sophisticated approach seems to be to estimate the phases in the above model by means of a particle filter. This has been done in Dahlhaus et al. (2017) in the univariate case $d=1$ [in the more general setting (i)-(iii) from below]. However, also with this approach the situation is not clear: A proper test for synchronization would then, for example, be a likelihood ratio test based on the original observations $Y_{t}$. The test statistic for such a test could be approximated by means of a particle filter but its distribution under the null hypothesis is also not clear and difficult to derive.

Our personal view is that for systems with stronger noise the above particle filter or a periodogram based method lead to better estimation results. For chaotic oscillators as in Section 5.1, the Hilbert transform seems to be the better choice. Furthermore, the Hilbert transform is used in most applications.

\section{Generalizations of the State Space Model}

(i) Other oscillation patterns:

For noncosine type signals, one may use instead the observation equation

$$
Y_{t}=a_{t} f\left(\phi_{t}\right)+b_{t}+\varepsilon_{t},
$$

with $f$ being a $2 \pi$-periodic real valued function representing the oscillation pattern (cf. Dahlhaus et al., 2017). $f$ typically is unknown and needs to be estimated. An example are ECG-data. In addition, one may need a time varying amplitude and a baseline. This can be achieved with a state space model also for $a_{t}$ and $b_{t}$, for example, a VAR-model with mean different from zero.

(ii) Positivity of the phase increments:

In nearly all cases, it is clear that the phase increments of an oscillator should be positive. However, in the above model with Gaussian noise also negative phase increments may occur. This happens with small probability since $\omega_{0}$ usually is large in comparison with the standard deviation of the $\eta_{t}$. Furthermore, this should not be a big problem if the model is mainly used for estimation and testing (and not for simulation).

To overcome this problem, we have used in Dahlhaus et al. (2017) in the case of a single oscillator an integrated ACD (autoregressive conditional duration) 
model for the state. At present, we are having no idea how this ACD-model can be extended to the cointegrated case (see, however, Mosconi and Olivetti, 2005, for bivariate ACD-models).

\section{APPENDIX B: APPENDIX 2: COMPUTATIONAL AND MODELING ASPECTS}

\section{B.1 Computational Aspects}

There exists several software programmes on cointegration analysis. For example, the CATS module within the RATS Econometrics Software (http://www. estima.com/) and OxMetrics (http://www.oxmetrics. net/). For the analysis of the Rössler-Lorenz system in Section 5.1, we have used the R-packages urca (http:// cran.r-project.org/web/packages/urca/index.html—see Pfaff, 2008) and systemfit (http://cran.r-project.org/ web/packages/systemfit/index.html; see Henningsen and Hamann, 2007). Here are some details:

\section{Initial situation:}

The unwrapped phases are given as vectors phi1, phi2 (e.g., determined by the Hilbert-transform). The first values phi1[1] and phi2[2] should both lie in $[0,2 \pi)$.

2. Johansen LR-test (top-bottom version):

require(urca)

joha.test <- ca.jo(cbind(phi1,phi2), type = "trace", ecdet = "none", $\mathrm{K}=2$, spec="transitory")

print(summary(joha.test))

Note: The specification ecdet $=$ "none" fits (9) with $\mu$, that is, with $\omega_{0}$ and $\beta_{0}$ (Case 3 in Juselius, 2006, Chapter 6.3) (while ecdet $=$ "const" and ecdet $=$ "trend" correspond to case 2 and case 4, resp.). We recommend using the quantiles from the Appendix of Juselius (2006) (Case 3) instead of the printed values in the summary.

2a. ADF-test for testing phase synchronization (testing the phase-difference for integrated process vs. stationary process):

require(urca)

beta $2<-1$ \# Specify cointegration vector beta adf.test $<-$ ur.df(phi1 - beta $2 *$ phi2, type="drift") print(summary(adf.test))

Note: The specification type $=$ "drift" corresponds to Case 3 in Hamilton (1994), page 529. The relevant statistics is tau2 which should be compared with the quantiles of the standard normal distribution (for sample sizes less than 300 , the t-distribution probably gives the better quantiles). The quantiles printed in the summary correspond to Case 2 in Hamilton (1994) where a different hypothesis is tested with the same statistics.
3. and 3a. Estimation, further testing and unidirectional coupling:

require(systemfit)

$\mathrm{T}<-$ length(phi1)

err.lag1 <- (phi1-beta2*phi2)[-c(T-1, T)]

err.lag2 <- (phi1-beta2*phi2)[-c(T-1, T)]

dphi $1<-\operatorname{diff}($ phi1)

dphi $2<-\operatorname{diff}($ phi2)

diff.dat <- data.frame(embed(cbind(dphi1, dphi2), 2))

colnames(diff.dat) <- c('dphi1', 'dphi2', 'dphi1.1', 'dphi2.1')

eqPhi $1<-$ dphi $1 \sim$ err.lag $1+$ dphi $1.1+$ dphi 2.1

eqPhi $2<-$ dphi $2 \sim$ err.lag $2+$ dphi2 $21+$ dphi1.1

system $<-$ list $($ phi1 = eqPhi1, phi2 = eqPhi2)

estSystem <- systemfit(system, data=diff.dat)

print(summary(estSystem)).

4. Final estimation of the reduced system:

eqPhi $1<-$ dphi $1 \sim$ dphi1.1

eqPhi $2<-$ dphi $2 \sim$ err.lag $2+$ dphi 2.1

system $<-$ list $($ phi $1=$ eqPhi 1, phi2 $=$ eqPhi2)

estReducedSystem <- systemfit(system, data=diff.dat) print(summary(estReducedSystem)).

\section{B.2 Modeling Aspects}

One needs to be aware of the fact that the VECmodel has been applied in Section 5.1 in the highly misspecified situation of two noisy chaotic oscillators. Our goal was to demonstrate that already a "simple" VEC-model can be used to successfully identify phase synchronization and unidirectional coupling. For that reason, we have chosen the fixed order $p=2$. In Figure 3 , the oscillation of the phase of the Lorenz attractor is due to the slightly steeper ascent than the descent of the Lorenz attractor. In an additional data analysis (not reported here), we have taken this into account by choosing the higher model order $p=4$ for the ARpart. This has led to a clear improvement of the fit for the Lorenz attractor while higher model orders than $p=4$ only showed smaller improvements. We therefore have redone the complete analysis of Section 5.1 with $p=4$. There was a clear quantitative improvement in the model fit but qualitatively all results stayed the same.

In Section 5.2, we have chosen the higher order $p=$ 6 due to the in-cycle fluctuation of the phases and the phase-differences. Here, the model order was chosen by inspection of the significant lags in the parametric model fit.

A common suggestion in time series analysis is to use a model selection criterion for choosing $p$ such as the AIC or the BIC (cf. Greene, 2008, page 752). In 
the case of the nonlinear Rössler-Lorenz system investigated in Section 5.1, we have found with the BIC the exorbitant model order $p=31$ and with the AIC even $p=95$ for the phases of the Lorenz attractor (although not all lags were significant). This reflects the fact that we need a very high order to obtain a good approximation of the misspecified system with a VEC-model. On the other hand, we would hardly trust the outcome of significance tests, etc. with such a large order. For that reason, one has to refrain from these automatic procedures for chaotic oscillators and look for a more parsimonious model order. Of course, this topic requires deeper investigation.

\section{ACKNOWLEDGMENTS}

This work was part of the project "Econometric Models for Phase Synchronization" supported by the University of Heidelberg under the grant Frontier D.801000/08.023 submitted in March 2008.

The first author had the privilege to be from 20012007 a member of the DFG-priority programme SPP 1114 "Mathematical methods for time series analysis and digital image analysis" (jointly with many physicists). In this joint project, he learned about phase synchronization from several colleagues including Jürgen Kurths, Ulrich Parlitz, Björn Schelter and Jens Timmer; see Dahlhaus et al. (2008).

We are grateful to the associate editor and Søren Johansen for very helpful comments, and to Florian Müller-Dahlhaus for extensive discussions on the role of phase synchronization in neuroscience.

\section{DISCLAIMER}

The views expressed here are those of the authors and not necessarily those of their employers.

\section{REFERENCES}

Allefeld, C. and KURTHS, J. (2004a). An approach to multivariate phase synchronization analysis and its application to eventrelated potentials. Internat. J. Bifur. Chaos Appl. Sci. Engrg. 14 417-426. MR2069592

Allefeld, C. and KuRThs, J. (2004b). Testing for phase synchronization. Internat. J. Bifur. Chaos Appl. Sci. Engrg. 14 405416. MR2069591

Banerjee, A., Galbraith, J., Dolado, J. and Hendry, D. (1993). Co-Integration, Error Correction, and the Econometric Analysis of Non-Stationary Data. Oxford Univ. Press, Oxford.

Baptista, M., Silva, T., Sartorelli, J., Caldas, I. and RosA, E. JR. (2003). Phase synchronization in the perturbed Chua circuit. Phys. Rev. E 67056212.
Blasius, B., Huppert, A. and Stone, L. (1999). Complex dynamics and phase synchronization in spatially extended ecological systems. Nature 399 354-359.

Boccaletti, S., Pecora, L. and Pelaez, A. (2001). Unifying framework for synchronization of coupled dynamical systems. Phys. Rev. E 63066219.

Boccaletti, S., Kurths, J., Osipov, G., Valladares, D. L. and ZHOU, C. S. (2002). The synchronization of chaotic systems. Phys. Rep. 366 1-101. MR1913567

BRILlinger, D. R. (2001). Time Series: Data Analysis and Theory. Classics in Applied Mathematics 36. SIAM, Philadelphia, PA. Reprint of the 1981 edition. MR1853554

Colgin, L. and Moser, E. (2010). Gamma oscillations in the hippocampus. Physiology 25 319-329.

Dahlhaus, R., Kurths, R., MaAs, P. and Timmer, J., eds. (2008). Mathematical Methods in Time Series Analysis and Digital Image Processing. Springer, Berlin and Heidelberg.

Dahlhaus, R., Dumont, T., Le CorfF, S. and NedderMEYER, J. C. (2017). Statistical inference for oscillation processes. Statistics 51 61-83. MR3600462

David, O., Cosmelli, D., LachauX, J.-P., Baillet, S., GarNERo, L. and MARTINERIE, J. (2003). A theoretical and experimental introduction to the non-invasive study of largescale neural phase synchronization in human beings. Internat. J. Comput. Cog. 1 53-77.

DeShazer, D. J., Breban, R., Ott, E. and Roy, R. (2001). Detecting phase synchronization in a chaotic laser array. Phys. Rev. Lett. 87044101.

DiCKEY, D. A. and Fuller, W. A. (1979). Distribution of the estimators for autoregressive time series with a unit root. J. Amer. Statist. Assoc. 74 427-431. MR0548036

DiCKEY, D. A. and Fuller, W. A. (1981). Likelihood ratio statistics for autoregressive time series with a unit root. Econometrica 49 1057-1072. MR0625773

Elson, R., Selverston, A., Huerta, R., Rulkov, N., RABINOVICH, M. and ABARBANEL, H. (1998). Synchronous behavior of two coupled biological neurons. Phys. Rev. Lett. 81 5692-5695.

Engel, A., Fries, P. and Singer, W. (2001). Dynamic predictions: Oscillations and synchrony in top-down processing. Nat. Rev., Neurosci. 2 704-716.

ENGLE, R. F. and GRANGER, C. W. J. (1987). Co-integration and error correction: Representation, estimation, and testing. Econometrica 55 251-276. MR0882095

Engle, R. and White, H. (1999). Cointegration, Causality and Forecasting. Oxford Univ. Press, Oxford.

FELL, J. and AXMACHER, N. (2011). The role of phase synchronization in memory processes. Nat. Rev., Neurosci. 12 105-118.

FUller, W. A. (1996). Introduction to Statistical Time Series, 2nd ed. Wiley, New York. MR1365746

Granger, C. W. J. (1981). Some properties of time series data and their use in econometric model specification. J. Econometrics 16 121-130.

Greene, W. (2008). Econometric Analysis, 6th ed. Pearson Prentice Hall, New Jersey.

Grossmann, A., Kronland-Martinet, R. and Morlet, J. (1989). Reading and understanding continuous wavelet transforms. In Wavelets, Time-Frequency Methods and Phase Space. Inverse Probl. Theoret. Imaging (J. Combes, ed.) 2-20. Springer, Berlin. MR1010896 
GuAn, S., LAI, C.-H. and WeI, G. W. (2005). Phase synchronization between two essentially different chaotic systems. Phys. Rev. E (3) 72 016205. MR2178376

Hamilton, J. D. (1994). Time Series Analysis. Princeton Univ. Press, Princeton, NJ. MR1278033

Hannan, E. J. (1973). The estimation of frequency. J. Appl. Probab. 10 510-519. MR0370977

Henningsen, A. and Hamann, J. D. (2007). systemfit: A package for estimating systems of simultaneous equations in $r$. J. Stat. Softw. 23 1-40.

Horvath, M. T. K. and Watson, M. W. (1995). Testing for cointegration when some of the cointegrating vectors are prespecified. Econometric Theory 11 984-1014. Trending multiple time series (New Haven, CT, 1993). MR1458947

JOHANSEN, S. (1991). Estimation and hypothesis testing of cointegration vectors in Gaussian vector autoregressive models. Econometrica 59 1551-1580. MR1135641

JOHANSEN, S. (1995). Likelihood-Based Inference in Cointegrated Vector Autoregressive Models. Oxford Univ. Press, New York. MR1487375

Juselius, K. (2006). The Cointegrated VAR Model: Methodology and Applications. Advanced Texts in Econometrics. Oxford Univ. Press, Oxford. MR2358946

Kammerdiner, A. R. and Pardalos, P. M. (2010). Analysis of multichannel EEG recordings based on generalized phase synchronization and cointegrated VAR. In Computational Neuroscience. Springer Optim. Appl. 38 317-339. Springer, New York. MR2656819

Kammerdiner, A., Boyko, N., Ye, N., He, J. and PardaLOS, P. (2010). Integration of signals in complex biophysical system. In Dynamics of Information Systems (M. Hirsch, P. Pardalos and R. Murphey, eds.) 197-211. Springer, New York. MR2572384

Kessler, M. and RAhbeK, A. (2001). Asymptotic likelihood based inference for co-integrated homogenous Gaussian diffusions. Scand. J. Stat. 28 455-470. MR1858411

KISS, I. Z. and HuDSON, J. L. (2001). Phase synchronization and suppression of chaos through intermittency in forcing of an electrochemical oscillator. Phys. Rev. E 64046215. DOI:10.1103/PhysRevE.64.046215.

KISS, I. and HUDSON, J. (2002). Phase synchronization of nonidentical chaotic electrochemical oscillators. Phys. Chem. Chem. Phys. 4 2638-2647.

KISS, I., LV, Q. and HudSON, J. (2005). Synchronization of nonphase-coherent chaotic electrochemical oscillations. Phys. Rev. E 71035201.

KocAReV, L. and PARlitz, U. (1996). Generalized synchronization, predictability, and equivalence of unidirectionally coupled dynamical systems. Phys. Rev. Lett. 76 1816-1819.

Kremers, J. J. M., ERICSSON, N. R. and Dolado, J. J. (1992). The power of cointegration tests. Oxf. Bull. Econ. Stat. 54 32548.

KuRAmoto, Y. (1975). Self-entrainment of a population of coupled non-linear oscillators. In Lecture Notes in Phys. 39 420422. Springer, Berlin. MR0676492

Kuramoto, Y. (1984). Chemical Oscillations, Waves, and Turbulence. Springer Series in Synergetics 19. Springer, Berlin. MR0762432

Kwiatkowski, D., Phillips, P., Schmidt, P. and Shin, Y. (1992). Testing the null hypothesis of stationarity against the alternative of a unit root: How sure are we that economic time series have a unit root? J. Econometrics 54 159-178.

LÜTKEPOHL, H. (2005). New Introduction to Multiple Time Series Analysis. Springer, Berlin. MR2172368

Maraun, D. and KuRThs, J. (2005). Epochs of phase coherence between El Niño/Southern Oscillation and Indian monsoon. Geophys. Res. Lett. 32. DOI:10.1029/2005GL023225.

Mormann, F., Lehnertz, K., David, P. and Elger C, E. (2000). Mean phase coherence as a measure for phase synchronization and its application to the EEG of epilepsy patients. Phys. D 144 358-369.

Mosconi, R. and OlivetTi, F. (2005). Bivariate generalizations of the ACD models. Presented at the Journal of Applied Econometrics Annual Conference, Venezia.

Osipov, G. V., KuRTHS, J. and ZHOU, C. (2007). Synchronization in Oscillatory Networks. Springer Series in Synergetics. Springer, Berlin. MR2350638

PAluš, M. and VeJMelKa, M. (2007). Directionality of coupling from bivariate time series: How to avoid false causalities and missed connections. Phys. Rev. E (3) 75 056211. MR2361831

PAluš, M., KomáreK, V., HrNČířr, Z. and ŠTĚrbová, K. (2001). Synchronization as adjustment of information rates: Detection from bivariate time series. Phys. Rev. E 63046211. DOI:10.1103/PhysRevE.63.046211.

PAlut, Y. and Zanone, P.-G. (2005). A dynamical analysis of tennis: Concepts and data. J. Sports Sci. 23 1021-1032.

PARAschakis, K. and DAhlhaus, R. (2012). Frequency and phase estimation in time series with quasi periodic components. J. Time Series Anal. 33 13-31. MR2877605

PeCorA, L. M. and CARroll, T. L. (1990). Synchronization in chaotic systems. Phys. Rev. Lett. 64 821-824. MR1038263

PFAFF, B. (2008). Analysis of Integrated and Cointegrated Time Series with R, 2nd ed. Use R! Springer, New York. MR2450313

Phillips, P. C. B. (1991). Error correction and long-run equilibrium in continuous time. Econometrica 59 967-980. MR1113542

Phillips, P. C. B. and Ouliaris, S. (1990). Asymptotic properties of residual based tests for cointegration. Econometrica $\mathbf{5 8}$ 165-193. MR1046923

Pikovsky, A., Rosenblum, M. and Kurths, J. (2001a). Synchronization: A Universal Concept in Nonlinear Sciences. Cambridge Nonlinear Science Series 12. Cambridge Univ. Press, Cambridge, MA. MR1869044

Pikovsky, A. S., Rosenblum, M. G., Osipov, G. V. and KURTHS, J. (1997). Phase synchronization of chaotic oscillators by external driving. Phys. D 104 219-238. MR1453087

Press, W. H., Teukolsky, S. A., Vetterling, W. T. and Flannery, B. P. (1992). Numerical Recipes in C, 2nd ed. Cambridge Univ. Press, Cambridge.

Pujol-Pere, A., Calvo, O., Matias, M. and Kurths, J. (2003). Experimental study of imperfect phase synchronization in the forced Lorenz system. Chaos 13 319-326.

Quian Quiroga, R., Kreuz, T. and Grassberger, P. (2000). Learning driver-response relationships from synchronization patterns. Phys. Rev. E 61 5142-5148.

Quian Quiroga, R., Kreuz, T. and Grassberger, P. (2002). Performance of different synchronization measures in real data: A case study on electroencephalographic signals. Phys. Rev. E 65 041903. DOI:10.1103/PhysRevE.65.041903. 
Rosenblum, M., Pikovsky, A. and Kurths, J. (1996). Phase synchronization of chaotic oscillators. Phys. Rev. Lett. 76. 1804-1807.

SAIKKONEN, P. and LÜTKEPOHL, H. (2000). Testing for the cointegrating rank of a VAR process with an intercept. Econometric Theory 16 373-406. MR1769280

Schelter, B., Winterhalder, M., Timmer, J. and PEIFER, M. (2007). Testing for phase synchronization. Phys. Lett. A 366 382-390.

StefanovsKA, A. (2002). Cardiorespiratory interactions. Nonlinear Phenom. Complex Syst. 5 462-469. MR2013768

Stefanovska, A., Haken, H., McClintock, P. V. E., Hožič, M., BAJrović, F. and RiBARIČ, S. (2000). Reversible transitions between synchronization states of the cardiorespiratory system. Phys. Rev. Lett. 85 4831-4834. DOI:10.1103/PhysRevLett.85.4831.

Strogatz, S. H. (2000). From Kuramoto to Crawford: Exploring the onset of synchronization in populations of coupled oscillators. Phys. D 143 1-20. Bifurcations, patterns and symmetry. MR1783382
Tass, P., Rosenblum, M. G., Weule, J., Kurths, J., Pikovsky, A., Volkmann, J., Schnitzler, A. and FreUND, H. J. (1998). Detection of $n: m$ phase locking from noisy data: Application to magnetoencephalography. Phys. Rev. Lett. 81 3291-3294.

Van Leeuwen, P., Geue, D., Thiel, D., Cysarz, D., Lange, S., Romano, M., Wessel, N., Kurths, J. and GRÖNEMEYER, D. (2009). Influence of paced maternal breathing on fetal-maternal heart rate coordination. In Proceedings of the National Academy of Sciences of the United States of America (PNAS) 106 13661-13666.

Varela, F., Lachaux, J.-P., Rodriguez, E. and MarTINERIE, J. (2001). The brainweb: Phase synchronization and large-scale integration. Nat. Rev. Neurosci. 2 229-239.

WinfreE, A. T. (1967). Biological rhythms and the behavior of populations of coupled oscillators. J. Theor. Biol. 16 15-42.

Womelsdorf, T., Schoffelen, J.-M., Oostenveld, R., Singer, W., Desimone, R., Engel, A. K. and Fries, P. (2007). Modulation of neuronal interactions through neuronal synchronization. Science 316 1609-1612. 